\title{
Unsteady Airloads on Static Airfoils through High Angles of Attack and in Reverse Flow
}

\author{
Andrew H. Lind ${ }^{1}$ and Anya R. Jones ${ }^{2}$ \\ Department of Aerospace Engineering, University of Maryland, College Park, 20742
}

\begin{abstract}
The present work is aimed at understanding the sources, magnitude, and frequency of unsteady airloads acting on airfoils at high angles of attack and in reverse flow in order to improve the design of rotor blades for high-speed helicopters and wind turbines. Four rotor blade airfoils were tested at angles of attack through $180^{\circ}$ and at three Reynolds numbers, up to one million. The unsteady airloads acting on each airfoil were calculated by integrating time-resolved pressure measurements acquired along the midspan of the airfoil. Unsteady velocity fields for a NACA 0012 were calculated from time-resolved particle image velocimetry measurements. For all airfoils, the unsteady airloads were found to be large in magnitude near stall, when an unstable shear layer induces unsteady flow on the suction side of the airfoil. At post-stall angles of attack, the unsteady airloads generally decreased as a region of separated flow builds over the suction side. As the angle of attack was increased further, the airloads become periodic and the flow entered the deep stall vortex shedding regime. At high angles of attack $\left(30^{\circ} \leq \alpha \leq 150^{\circ}\right)$, the unsteady airloads were greatest for airfoils with a blunt aerodynamic trailing edge due to unsteady induced flow from trailing edge vortices. Aerodynamic hysteresis was shown to cause large unsteady airloads as the static angle of attack is decreased near stall. The unsteady airloads of a NACA 0012 in reverse flow were observed to be insensitive to Reynolds number due to flow separation at the sharp leading edge of this relatively thin airfoil.
\end{abstract}

Keywords: Reverse flow, Unsteady airloads, Vortex shedding, Leading edge separation, Shear layer

\footnotetext{
${ }^{1}$ Corresponding author. Graduate Research Assistant. 1151 Manufacturing Bldg., College Park, MD 20742. (508) 237-0599. alind@umd.edu

${ }^{2}$ Assistant Professor. 3154 Glenn L. Martin Hall, College Park, MD 20742. arjones@umd.edu
} 


\section{Introduction}

The rotor blades of high-speed helicopters and wind turbines often operate at high angles of attack $\left(30^{\circ} \leq \alpha \leq 150^{\circ}\right)$ leading to massive flow separation, unsteady airloads $\left(c_{l}, c_{d}\right.$, and $c_{m}$ ), and vibrations. High angle of attack flow occurs during start-up or stopping of a wind turbine when the rotational velocity is low $[1,2]$. It can also occur on the retreating blade of a helicopter in forward flight when the in-plane velocity component reaches zero and only inflow contributes to the local flow velocity [3]. A sizable portion of helicopter rotor blades can also operate in reverse flow, where flow travels from the geometric trailing edge towards the geometric leading edge. The reverse flow region exists on the retreating side of a rotor disk and grows in size with advance ratio, $\mu=U_{\infty} / \Omega R$. Up to $80 \%$ (or more) of the span of retreating rotor blades on a modern high-speed helicopter can be affected by reverse flow [4].

The aerodynamic environment of rotor blades features additional flow complexities such as unsteady airfoil oscillations (e.g., cyclic pitching), three-dimensionality (e.g., radial flow), and a time-varying local freestream. These aspects of the flow, combined with high angle of attack and reverse flow, contribute to unsteady airloads experienced by a rotor blade. Some studies have investigated the unsteady pressure distribution on rotor blades in the reverse flow region, but the magnitude of unsteady airloads in these flow regimes is still largely unknown $[5,6,7,8]$.

The aim of the present work is to experimentally study the unsteady airloads on a fundamental model of high angle of attack and reverse flow conditions: a static, two-dimensional airfoil held at angles of attack through $180^{\circ}$ while subjected to a constant freestream. Unsteady airloads are calculated through integration of time-resolved surface pressure measurements. The effects of trailing edge shape, thickness, and camber on unsteady airloads are investigated. Reynolds number effects are also considered to account for the wide range of flow velocities encountered in the reverse flow region of a rotor [9]. The insight gained here 
will aid in understanding some of the sources of vibrations of rotor blades on wind turbines and high-speed helicopters operating at high angles of attack.

\section{Background}

Most prior work concerning airfoils at high angles of attack and in reverse flow has primarily focused on the time-averaged aerodynamics $[9,10,11,12,13,14,15,16]$. After the lift peak just prior to stall, a second lift peak occurs near $\alpha \approx 45^{\circ}$ due to a buildup of high pressure on the pressure side of the airfoil. Maximum drag occurs near $\alpha \approx$ $90^{\circ}$, when the airfoil is oriented perpendicular to the freestream. In reverse flow (focusing on $0^{\circ} \leq-\alpha_{\text {rev }} \leq 30^{\circ}$, where $\alpha_{\text {rev }} \equiv \alpha-180^{\circ}$ ), the static stall characteristics of an airfoil strongly depend on the shape of its geometric trailing edge. Conventional airfoils with a sharp geometric trailing edge exhibit a thin airfoil stall in reverse flow; a region of separated flow originates from the sharp aerodynamic leading edge and grows over an increasing portion of the airfoil with increasing angle of attack. This behavior, combined with flow separation at the blunt aerodynamic trailing edge, results in drag that is at least twice as large as in forward flow (for $|\alpha| \leq 10^{\circ}$ ) [3, 15]. Airfoils with a blunt geometric trailing edge delay massive flow separation in reverse flow, but have greater downward-acting lift and pitching moment [15]. The time-averaged airloads of thick airfoils (in both forward and reverse flow) have also been found to vary with Reynolds number in the range $1.1 \times 10^{5} \leq R e \leq 6.6 \times 10^{5}$ due to sensitivity of the position and extent of laminar separation bubbles [9].

The unsteady aerodynamics of wings and airfoils fixed at high angles of attack and in reverse flow has also been investigated, but in less depth than time-averaged aerodynamics. Work has been conducted experimentally $[17,18]$ and by numerical simulation $[2,19,20]$. The unsteady wake of an airfoil in reverse flow can be categorized into one of three flow regimes [18]. The first regime, slender body vortex shedding, occurs at low angles of attack and is characterized by bluff body vortex shedding from a blunt aerodynamic trailing edge. 
This regime is believed to only exist for low Reynolds numbers, $R e \leq 1.65 \times 10^{5}$. The second regime, the turbulent wake regime, exists at greater Reynolds numbers (or at moderate reverse flow angles of attack, typically $6^{\circ} \leq-\alpha_{\text {rev }} \leq 12^{\circ}$ ). The turbulent wake regime has no periodicity. For conventional airfoils with a sharp geometric trailing edge, this regime occurs during the progression of thin airfoil stall as the region of separated flow grows. For airfoils with a blunt leading and trailing edge, this regime occurs at angles of attack where the flow is attached over much of the airfoil. The third regime is deep stall vortex shedding. This regime is characterized by massive flow separation and bluff body vortex shedding on a larger scale than slender body vortex shedding. The dominant periodic flow structures are a series of vortices that shed from the blunt aerodynamic trailing edge. These vortices induce flow near the surface of the suction side of the airfoil and are believed to be the dominant source of unsteady airloads in this regime.

Time-averaged pressure distributions have been measured on airfoils in forward flow since the 1920's [21], though most studies have only considered angles of attack just past stall. However, Naumann studied the pressure distribution of airfoils in reverse flow $\left(170^{\circ} \leq\right.$ $\alpha \leq 210^{\circ}$ )[22] and Pope measured pressure distributions at angles of attack through $180^{\circ}$, resulting in important fundamental findings [11]. For example, Pope's work shows that the center of pressure on the pressure side of the airfoil shifts from the leading edge towards the trailing edge as the angle of attack passes through $90^{\circ}$. This creates a large pitching moment as the shift in center of pressure increases the moment arm about the geometric quarter-chord. Furthermore, at these high angles of attack, the flow is massively separated. Flow separation leads to unsteady forcing on airfoils, a phenomenon known as buffeting [23]. This forcing can exist over a wide frequency band (i.e., aperiodic) or a narrow frequency band in the presence of vortex shedding (i.e., periodic). An understanding of buffeting, and unsteady airloads in general, is important for predicting structural response. For example, if the vortex shedding frequency matches a structural resonance frequency, the unsteady 
periodic forcing can induce vibrations (i.e., vortex induced vibrations) [24].

Unsteady airloads can be measured using direct time-resolved force measurements, but this typically requires a dynamic calibration of the force balance system to separate the structural and aerodynamic responses to unsteady aerodynamic forcing. Direct force measurements measure global loads acting on an entire model and do not allow for localization of sources of unsteady loading at specific regions along the chord. Time-resolved (i.e., unsteady) surface pressure measurements, however, provide a means to study the separated (and separating) flow characteristics and can be integrated to determine the impact of the flow features on the unsteady airloads. For example, unsteady surface pressure measurements have been used to study airfoil buffeting and the development of static stall $[25,26]$. In these studies, fluctuations in lift and pitching moment were found to be greatest post-stall $\left(\alpha=15^{\circ}\right)$ and decrease in magnitude as angle of attack is increased $\left(\alpha=20^{\circ}\right)[26]$.

In summary, the time-averaged aerodynamics of static airfoils at high angles of attack and in reverse flow has been studied extensively. More recently, the associated unsteady wake regimes and vortex shedding frequencies have also been characterized. However, little work exists that investigates the post-stall unsteady airloads of static airfoils, and a need remains for an improved understanding of the unsteady airloads of blades at high angles of attack and in reverse flow. Specifically, the current work aims to characterize the impact of previously identified unsteady wake regimes and bluff body vortex shedding behavior on the unsteady airloads. The static airfoil model presented here is a simplification of the true complex flow environment encountered by rotor blades that provides fundamental insight on the sources, magnitude, and frequency of unsteady airloads due to bluff-body-like behavior towards improving the design of rotor blades for wind turbines and high-speed helicopters. 


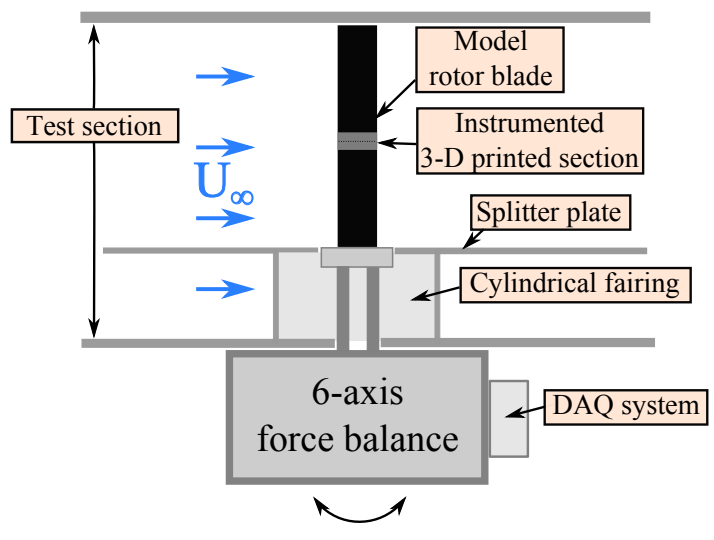

(a) Schematic of a model rotor blade mounted on the force balance.

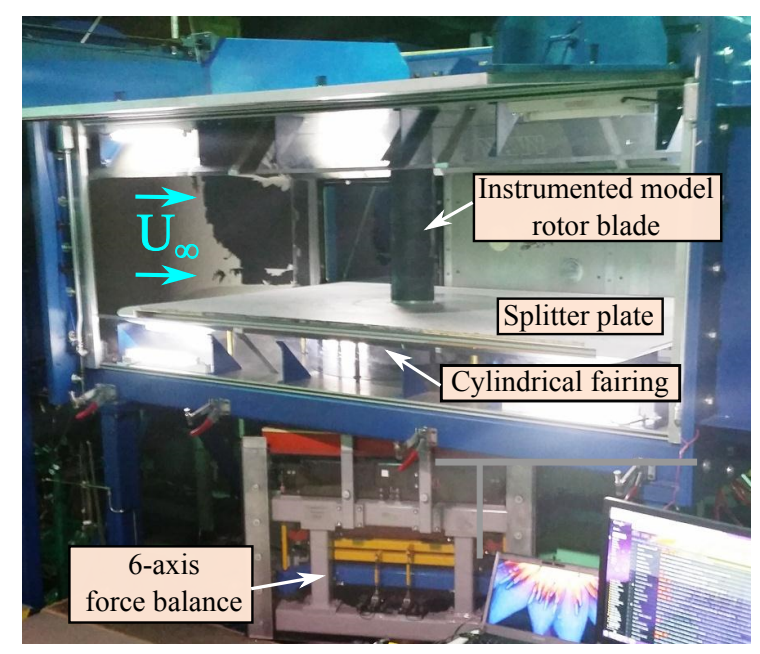

(b) Photograph of the test section with a model rotor blade installed.

Figure 1: Experimental set-up in the USNA $42 \times 60$ in wind tunnel [9].

\section{Methodology}

\subsection{Overview}

Wind tunnel testing was carried out at the United States Naval Academy (USNA) in a $42 \times 60$ in closed-circuit low-speed wind tunnel with a maximum speed of $200 \mathrm{mph}$. Figure 1 shows the experimental set-up. Four model rotor blades were fabricated from basswood, steel spars, and 3-D printed components. Each model had a span of 30.875 in and a chord of 8 in $(A R=3.86)$. Figure $1(\mathrm{a})$ shows a schematic of a model rotor blade mounted to the 6-axis force balance. Each model spanned the entire distance between a splitter plate and the test section ceiling. The splitter plate was installed to reduce the span of the model rotor blade, thereby reducing the maximum bending moment acting on the force balance. A gap of 0.125 in $(0.0156 c)$ existed between the top of the model and the test section ceiling to prevent interference with the force measurements. Figure 1(b) shows a photograph of the setup with the model blade mounted in a cantilevered configuration on the force balance. A cylindrical fairing was installed around the force balance mount to shield the mounts from the freestream. 


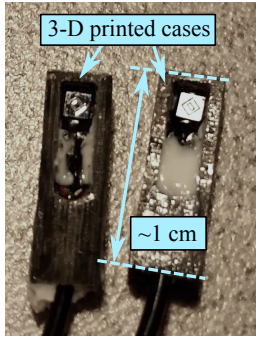

(a) Unsteady pressure transducers.

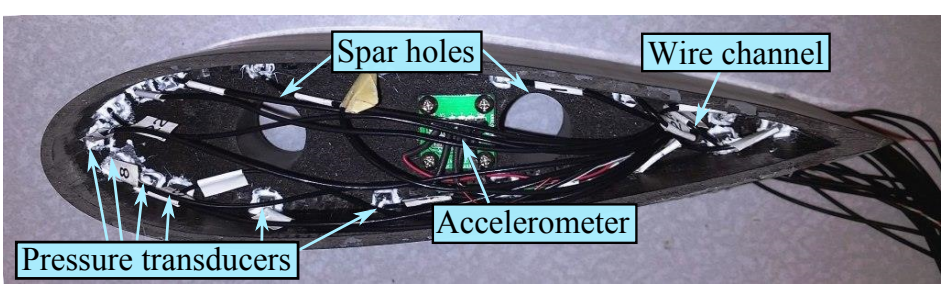

(b) Instrumented 3-D printed airfoil section (NACA 0024).

Figure 2: Model rotor blade instrumentation.

Each model rotor blade featured a 3-D printed section positioned at the mid-span that housed up to 18 unsteady pressure transducers. Figure 2 shows the instrumentation of a NACA 0024 airfoil section. Each pressure transducer (Kulite LL-3-072-25A, range: 025 psia) was potted into a 3-D printed case (Figure 2(a)). This design allowed the transducer/case units to be modular; they could be mounted and remounted in different model rotor blades. Figure 2(b) shows the pressure transducer units mounted along the inner surface of a NACA 0024 3-D printed airfoil section. Pressure taps with a diameter of 0.03 in were included in the design of these sections, positioned along a single spanwise location. Each transducer/case unit was sealed with silicon RTV. The transducer wires were channeled through the model rotor blade to a 22-bit data acquisition system located outside the force balance (Figure 1(a)). Time-resolved pressure measurements were captured at a sampling rate of $2 \mathrm{kHz}$, allowing for frequency content up to $1 \mathrm{kHz}$ to be detected. Measurements were collected for 5 seconds at each angle of attack. Time-averaged force balance measurements were also collected during this time and are the subject of prior work [9].

Figure 3 shows the airfoil profiles of the four model rotor blades considered in the present work. Two airfoils feature a sharp geometric trailing edge (NACA 0012 and NACA 0024) and two feature a blunt geometric trailing edge (elliptical and cambered elliptical). The latter two airfoils were selected to be representative of those found on modern high-speed helicopter 
rotor blades. These blunt, thick airfoils are often found near the blade root to mitigate flow separation and increase bending stiffness. The cambered elliptical airfoil $(26 \%$ thick, $4 \%$ camber) is similar to the publicly available Sikorsky DBLN-526 airfoil. Figure 3 also shows the locations of the pressure transducers along the blade surfaces. Pressure transducers that either had excessively high noise or an improper seal were deemed "inoperational"; these transducers were omitted from all analyses.

Geometry constraints prevented the installation of pressure transducers near the thinnest portions of the airfoils with a sharp trailing edge. The pressure on the upper and lower surface at $x / c=0.99$ as well as $x / c=1$ was therefore linearly extrapolated to allow for a more accurate integration of the pressure distribution when calculating airloads. The implementation of this extrapolation is shown in green in Figure 4. The time-averaged pressure distributions (including the extrapolated region) are compared with measurements from Pope [11]. Note that the results from the present work are for a NACA 0012 airfoil at $R e=6.6 \times 10^{5}$ while the results from Pope are for a NACA 0015 airfoil at $R e=1.23 \times 10^{6}$. Figure 4(a) shows good agreement between the results of the present work and Pope at $\alpha=10^{\circ}$. However, the lower spatial resolution of the pressures transducers in the present work fail to completely capture the pressure and suction peaks near the leading edge $(x / c=$ 0 ). The linear extrapolation also provides reasonable estimates for the pressure near the geometric trailing edge at higher angles of attack (Figures 4(b)-4(d)).

Time-resolved pressure measurements were collected for the four airfoils shown in Figure 3 at three Reynolds numbers: $3.3 \times 10^{5}, 6.6 \times 10^{5}$, and $1.0 \times 10^{6}$. For $R e=3.3 \times 10^{5}$ and $6.6 \times 10^{5}$, measurements were collected over $0^{\circ} \leq \alpha \leq 180^{\circ}$. The angle of attack was increased and decreased through static stall to capture aerodynamic hysteresis. For $R e=1.0 \times 10^{6}$, measurements were collected for $0^{\circ} \leq \alpha \leq 30^{\circ}$ (forward flow) and $150^{\circ} \leq \alpha \leq 180^{\circ}$ (reverse flow) with increasing magnitude of angle of attack only. The angle of attack resolution was $1^{\circ}$ during pre-stall conditions, $0.5^{\circ}$ near stall, $1^{\circ}$ post-stall, and $3^{\circ}$ for high angles of 

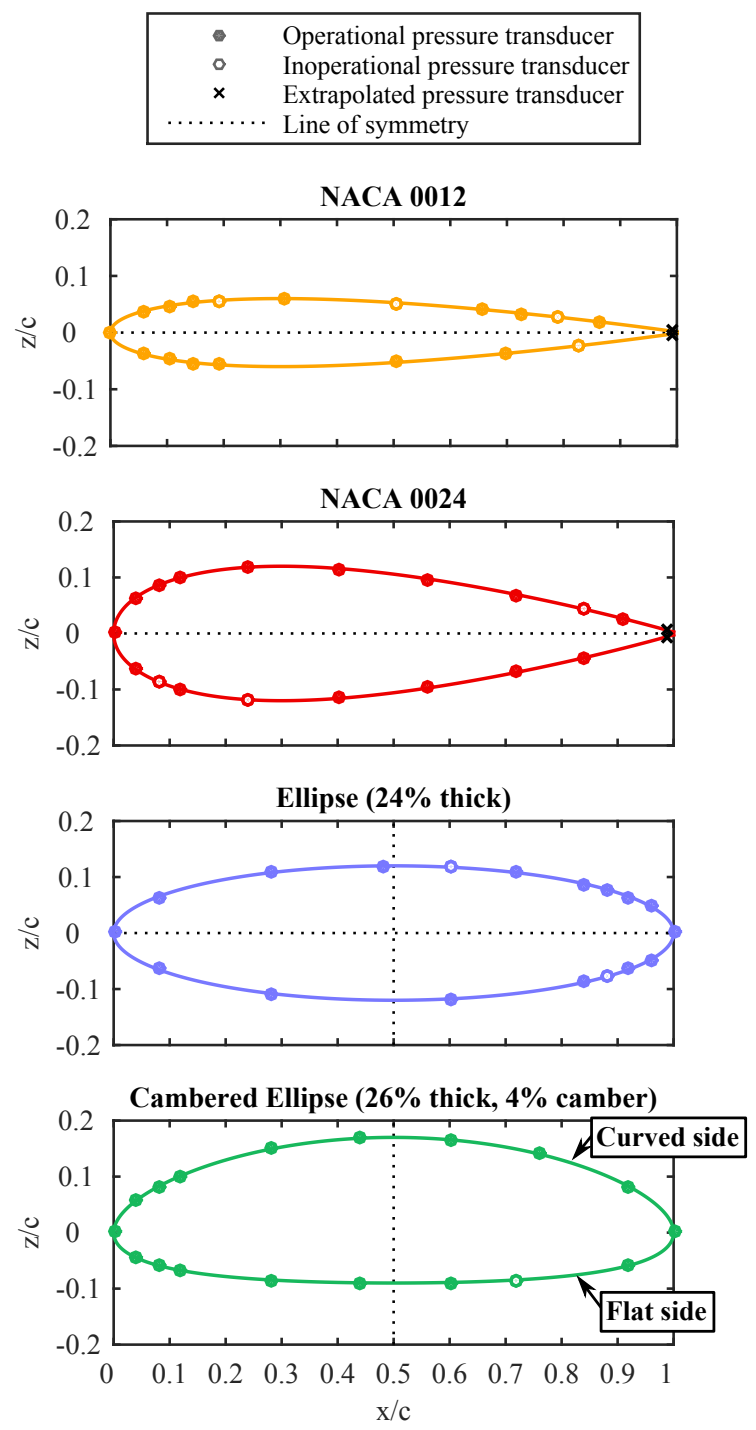

Figure 3: Profiles of airfoil sections tested in the present work. 


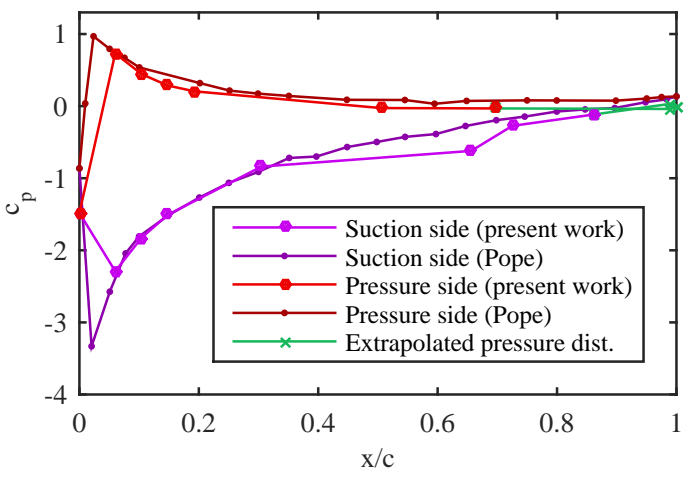

(a) $\alpha=10^{\circ}$.

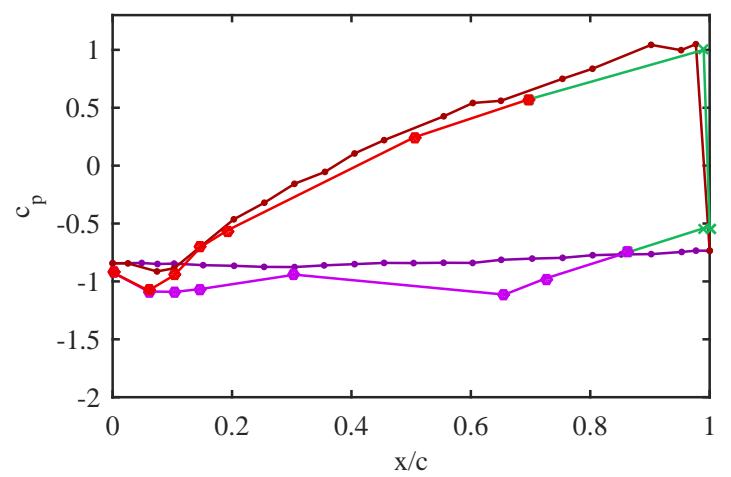

(c) $\alpha=150^{\circ}$.

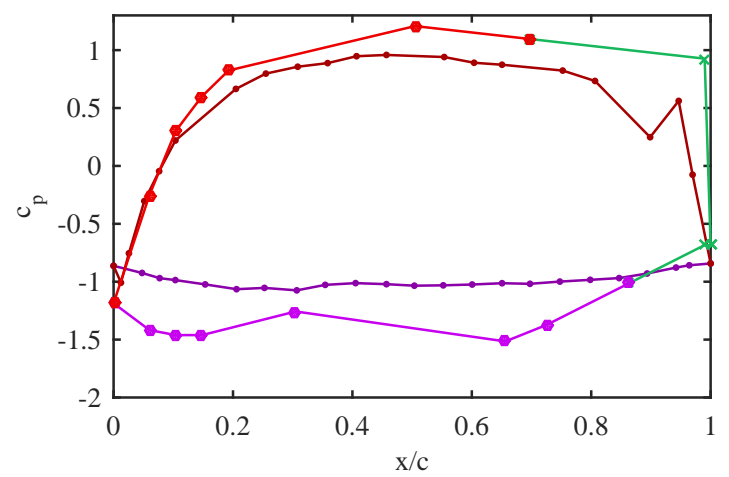

(b) $\alpha=90^{\circ}$.

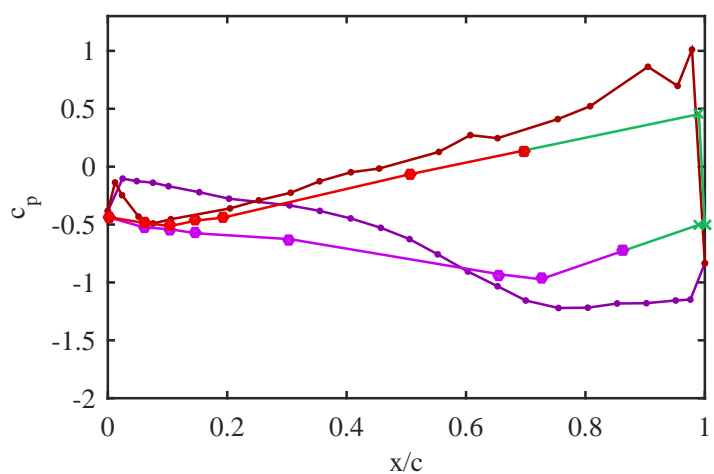

(d) $\alpha=170^{\circ}$.

Figure 4: Comparison of time-averaged pressure measurements in the present work (NACA 0012 at $R e=$ $\left.6.6 \times 10^{5}\right)$ with prior work by Pope [11] (NACA 0015 at $\left.R e=1.23 \times 10^{6}\right)$. 
attack (typically $30^{\circ} \leq \alpha \leq 150^{\circ}$ ). Note that at these high angles of attack, the model rotor blades were pinned to the ceiling of the wind tunnel test section to prevent excessive model vibrations. In a separate test, the models were left unpinned at $R e=3.3 \times 10^{5}$ for comparison with the pinned measurements. While contaminating the force balance measurements, pinning the models did not have a significant effect on either the time-averaged or unsteady pressure measurements for the full angle of attack range, $0^{\circ} \leq \alpha \leq 180^{\circ}$. As the airfoil incidence angle was varied during testing, the wind tunnel fan speed was adjusted to maintain constant dynamic pressure within the test section $( \pm 1 \%)$. Solid blockage was at a maximum $(13.3 \%)$ at $\alpha=90^{\circ}$; all results presented here are left uncorrected for solid blockage and wall effects.

In addition to the pressure and force measurements gathered in the USNA wind tunnel on instrumented models, time-resolved flow field measurements were collected on uninstrumented models using time resolved particle image velocimetry. These measurements were collected using a $20 \times 28$ in wind tunnel at the University of Maryland. Details of the setup and equipment can be found in Reference [15].

\subsection{Data Post-Processing}

\subsubsection{Pressure Integration Technique}

The magnitude of the time-averaged and unsteady airloads acting on each airfoil was computed from raw time-resolved pressure data. Figure 5 illustrates the panel technique used to integrate the surface pressure measurements. The NACA 0012 airfoil is shown for illustrative purposes. The integration approach used here determines discrete, local values of normal and tangential force components $\left(\Delta c_{n}\right.$ and $\left.\Delta c_{a}\right)$ using airfoil geometry and unsteady or time-averaged pressure measurements. These force components are then summed over the entire surface of the airfoil and transformed into airloads.

Panels were defined to sequentially connect the geometric coordinates of the operational and extrapolated pressure transducers in a closed path around each airfoil. The inset of 


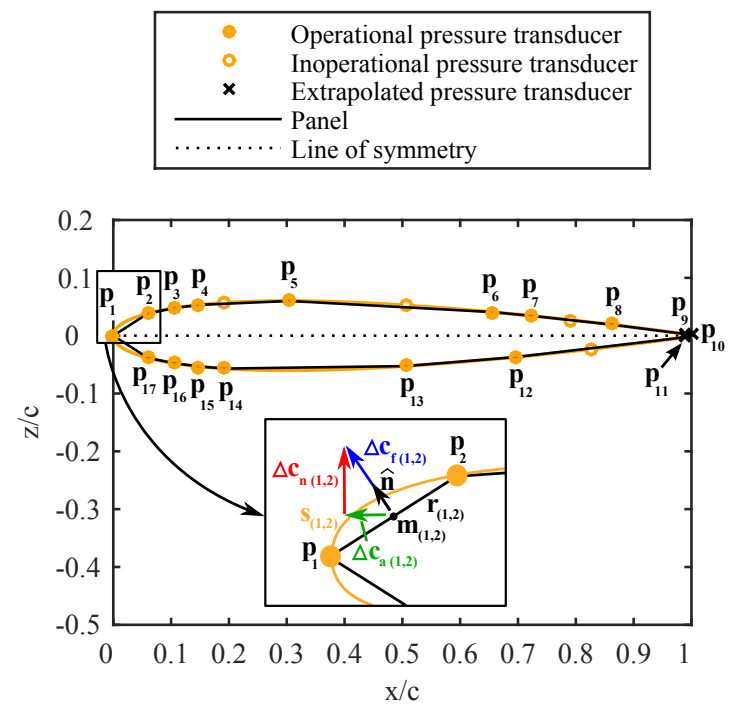

Figure 5: Panel technique used to calculate airloads (NACA 0012).

Figure 5 shows details of the panel between stations 1 and 2 on the NACA 0012. It is assumed that pressure varies linearly between any two stations, hence the pressure acting on this panel is $c_{p(1,2)}$ (the average of $p_{1}$ and $p_{2}$ ). The force acting on this panel, $\Delta c_{f(1,2)}$ could be found by integrating over the length of the panel, $r_{(1,2)}$. It is more accurate, however, to assume that the local pressure acts over the actual distance along the airfoil curvature, $s_{(1,2)}$. Hence, the local normal and tangential force components were found using:

$$
\begin{aligned}
& \Delta c_{n(1,2)}=-c_{p(1,2)} s_{(1,2)} \sin \theta \\
& \Delta c_{a(1,2)}=-c_{p(1,2)} s_{(1,2)} \cos \theta
\end{aligned}
$$

where $\theta$ is defined as the angle between the positive $x$-axis and the local unit normal vector, $\hat{n}$. The negative signs provide proper direction of the local force components. It is assumed that the force components act at the midpoint of each panel (e.g., $m_{(1,2)}$ in the inset of Figure 5). The coordinates of the panel midpoints were used in conjunction with $\Delta c_{n}$ and $\Delta c_{a}$ to calculate the local pitching moment, $\Delta c_{m}$, about the geometric quarter-chord. 
Finally, the airloads were calculated by summing over all $N$ panels:

$$
\begin{aligned}
c_{n} & =\sum_{i=1}^{N-1} \Delta c_{n(i, i+1)}+\Delta c_{n(N, 1)} \\
c_{a}= & \sum_{i=1}^{N-1} \Delta c_{a(i, i+1)}+\Delta c_{a(N, 1)} \\
c_{l}= & c_{n} \cos \alpha-c_{a} \sin \alpha \\
c_{d}= & c_{n} \sin \alpha+c_{a} \cos \alpha \\
c_{m}= & \sum_{i=1}^{N-1} \Delta c_{m(i, i+1)}+\Delta c_{m(N, 1)}
\end{aligned}
$$

It is important to note that the drag calculated in Equation 6 is the pressure drag and excludes skin friction drag.

\subsubsection{Magnitude of Unsteady Airloads}

Time-resolved airloads were calculated using the time-resolved pressure measurements (sampled at $2 \mathrm{kHz}$ ) for each airfoil at each angle of attack and Reynolds number. Figure 6(a) shows sample time traces (one-tenth of the total measurement sampling time) of the calculated time-resolved lift at three angles of attack for the NACA 0012 airfoil in forward flow. In all three cases, the black dashed line is positioned at the time-averaged lift value and the bounds of the shaded regions are two standard deviations $( \pm 2 \sigma)$, thereby capturing $95 \%$ of the fluctuations in lift. At $\alpha=2^{\circ}$ (blue), the time-averaged lift value is near 0.2 and the $2 \sigma$-variation is approximately 0.03 ; this value is small relative to the other two shaded regions shown in Figure 6(a). At this low angle of attack, the flow is attached and steady leading to small lift fluctuations. At $\alpha=14^{\circ}$ (red), the time-averaged lift is greater (nearly 0.6 ), but the $2 \sigma$-variation is also much greater than at $\alpha=2^{\circ}$ (nearly 0.2 ). At this angle of attack, the airfoil is stalled, leading to larger fluctuations in lift. 


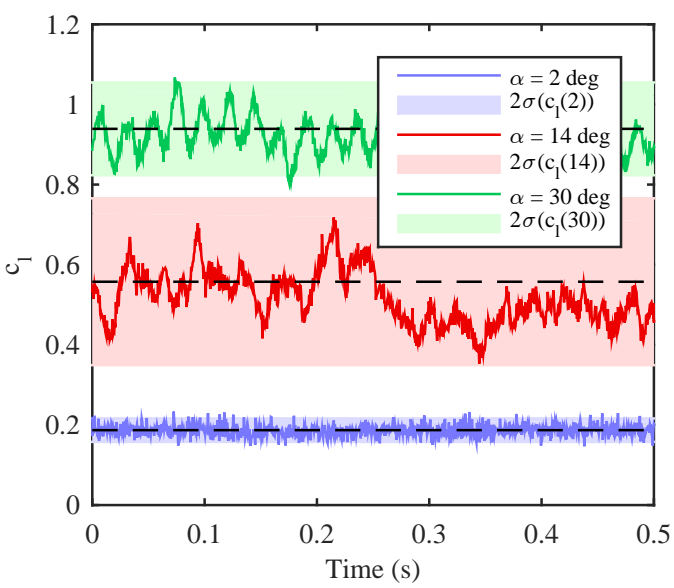

(a) Time trace.

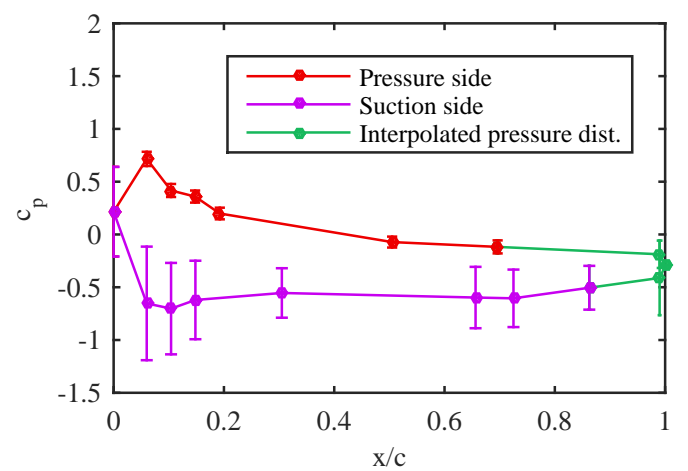

(c) Pressure distribution at $\alpha=14^{\circ}$. The error bars represent $2 \sigma\left(c_{p}\right)$.

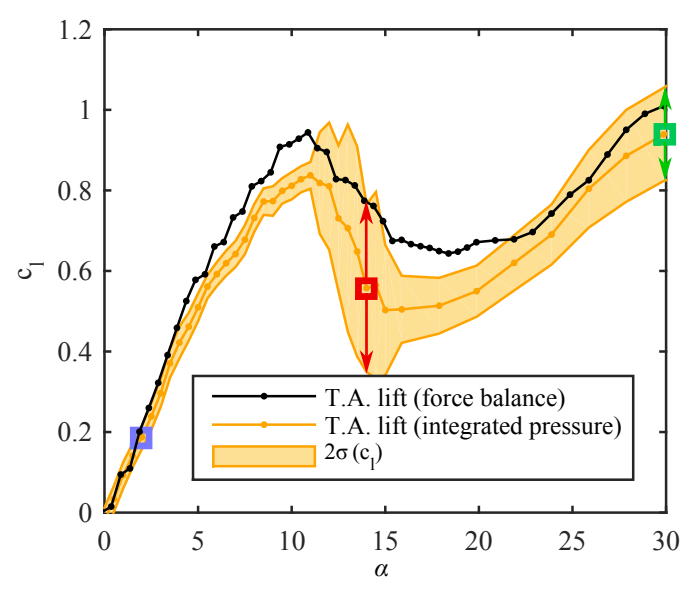

(b) Lift curve in forward flow.

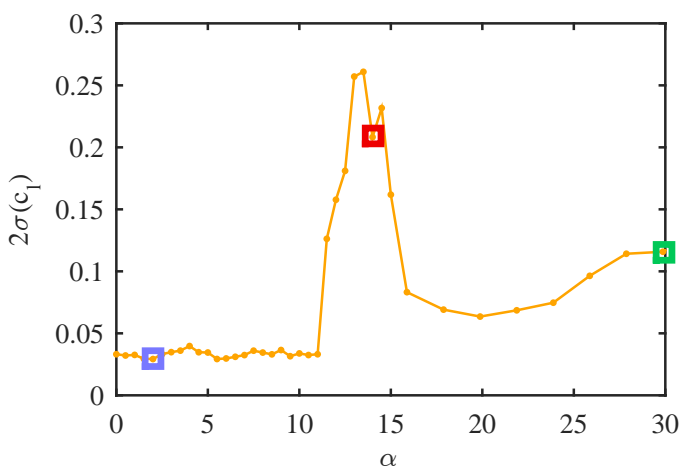

(d) Unsteady Lift.

Figure 6: Calculation of the magnitude of unsteady lift (NACA 0012 at $R e=3.3 \times 10^{5}$ ). 
Shifting attention to Figure 6(b), the time-averaged lift measured using the 6-axis force balance is compared with time-averaged lift calculated from integrating time-averaged pressure measurements. Note that the square symbols in Figure 6(b) are aligned horizontally with the black dashed lines in Figure 6(a). Similar to Figure 6(a), the shaded region surrounding the time-averaged lift curve (calculated from integrated pressure measurements) also represents the $2 \sigma$-variation of lift. For example, the arrows extending from the square symbol at $\alpha=14^{\circ}$ illustrate that the height of this filled region is equal to the height of the corresponding filled region of Figure 6(a). To examine the source of lift fluctuations more closely, Figure 6(c) shows the pressure distribution at $\alpha=14^{\circ}$. The error bars in this figure represent the local $2 \sigma$-variation of pressure fluctuations, $2 \sigma\left(c_{p}\right)$. It can be seen that the time-averaged pressure distribution along the suction side of the airfoil is nearly flat (typical for a stalled airfoil) while the pressure fluctuations are large due to the unsteadiness of the separated flow. This contrasts with the pressure side of the airfoil; the flow is attached here leading to a non-zero pressure gradient and small pressure fluctuations. The value of $2 \sigma\left(c_{l}\right)$ (the height of the filled regions in Figures 6(a) and 6(b)) is plotted against angle of attack in Figure 6(d). Here, it can be seen that $2 \sigma\left(c_{l}\right)$ is small for $0^{\circ} \leq \alpha \leq 11.5^{\circ}$ (attached flow regime) and increases sharply post-stall. From here on, quantities such as $2 \sigma\left(c_{l}\right)$ will be referred to as unsteady lift as it represents the range of lift fluctuations for a given set of flow conditions. The unsteady drag and pitching moment were calculated using the same approach illustrated in Figure 6. The time-averaged airloads will be referred to with $c_{l}, c_{d}$, and $c_{m}$.

\subsubsection{Convergence Study}

The flow over stalled airfoils at high angles of attack and in reverse flow is unsteady and can be either aperiodic or periodic [18]. In light of this unsteady behavior, it is important to verify that values calculated from time-resolved pressure measurements (e.g., time-averaged and unsteady pressures and airloads) have reached a reasonable convergence. To this end, 

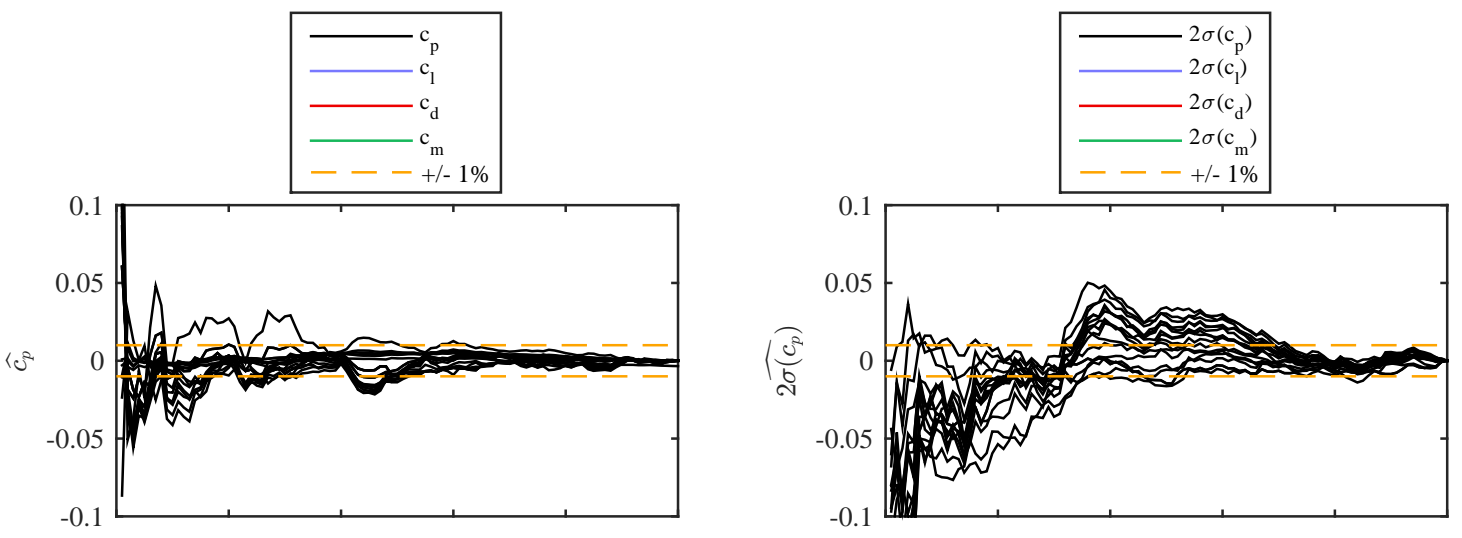

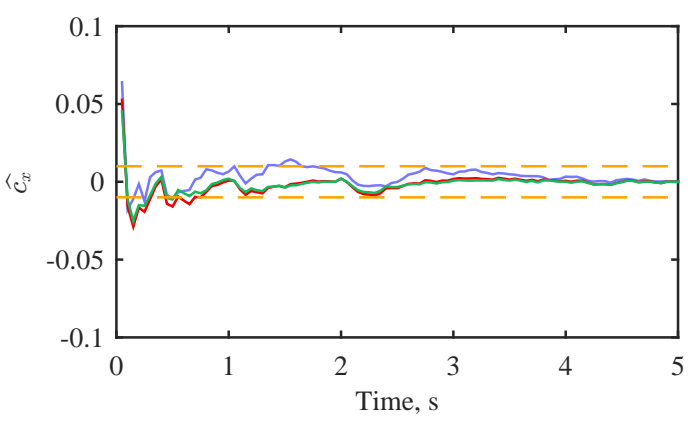

(a) Time-Averaged.

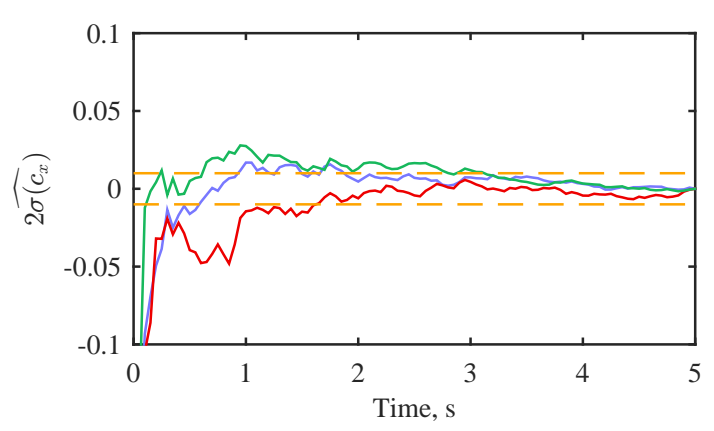

(b) Unsteady.

Figure 7: Sample results from a convergence study on the time-averaged and unsteady pressure measurements (upper plots) and airloads (lower plots). The case shown is for the NACA 0012 airfoil at $\alpha=90^{\circ}$ and $R e=6.6 \times 10^{5}$.

a convergence study was performed for a NACA 0012 airfoil operating at several angles of attack between $0^{\circ}$ and $180^{\circ}$. Figure 7 shows the results of this study at $\alpha=90^{\circ}$. In this orientation, a NACA 0012 airfoil behaves like a two-dimensional flat plate in a cross flow. The flow here is massively separated, leading to periodic bluff body vortex shedding in the wake [27]. This angle of attack was selected as an example to show here since it represents a "worst case" in terms of unsteadiness in the flow.

Figure 7(a) shows the convergence of time-averaged pressure measurements and calculated airloads. Focusing on the upper plot, the value of $\widehat{c_{p}}$ is plotted against time for each of the 17 pressure transducers (operational and extrapolated), where the $\widehat{(\cdot)}$ notation implies a percentage deviation from the average value calculated using the entire duration of the 
measurement:

$$
\widehat{c_{p}}=c_{p}(t) / c_{p}\left(t_{\max }\right)-1
$$

For the present work, $t_{\max }=5 \mathrm{~s}$. It can be seen that after $3.15 \mathrm{~s}$, the time-averaged pressure measurements converge to within $1 \%$ of the average pressure calculated for the full $5 \mathrm{~s}$ measurement duration. The time-averaged airloads calculated from the pressure measurements converge within $1.65 \mathrm{~s}$. Figure $7(\mathrm{~b})$ shows the convergence of the of unsteady pressures and airloads as defined in Section 3.2.2. In general, it can be seen that unsteady quantities in Figure 7(b) converge more slowly than time-averaged quantities (Figure 7(a)). Despite this, the unsteady pressure, $2 \sigma\left(c_{p}\right)$, still converges for all pressure transducers after $4.3 \mathrm{~s}$, while the unsteady airloads converge after $3.15 \mathrm{~s}$. The total sampling time of $5 \mathrm{~s}$ is therefore sufficient. The results of this convergence study are representative of other flow conditions.

\subsubsection{Identification of Vortex Shedding Frequencies}

In addition to quantifying the magnitude of unsteady airloads, the frequency content was also calculated. An $n$-point FFT was performed using the time-resolved lift signals (such as those presented in Figure 6(a)) to determine the frequency content with $0.1 \mathrm{~Hz}$ resolution. Figure 8(a) shows the frequency content of the lift signal for the NACA 0012 at $\alpha=150^{\circ}$, revealing a dominant peak at $41.6 \mathrm{~Hz}$. This dominant frequency (if present) was determined for each angle of attack from $0^{\circ} \leq \alpha \leq 180^{\circ}$. Figure 8(b) shows a nondimensionalized form of this dominant frequency in reverse flow $\left(150^{\circ} \leq \alpha \leq 180^{\circ}\right)$ using $S t_{d}=f d / U_{\infty}$, where $d$ is the projected diameter of the airfoil for a given angle of attack (see inset). This dominant frequency is plotted with known vortex shedding frequencies from flow field measurements [18], confirming that the dominant frequency in the time-resolved lift measurements corresponds to vortex shedding in the flow field. 


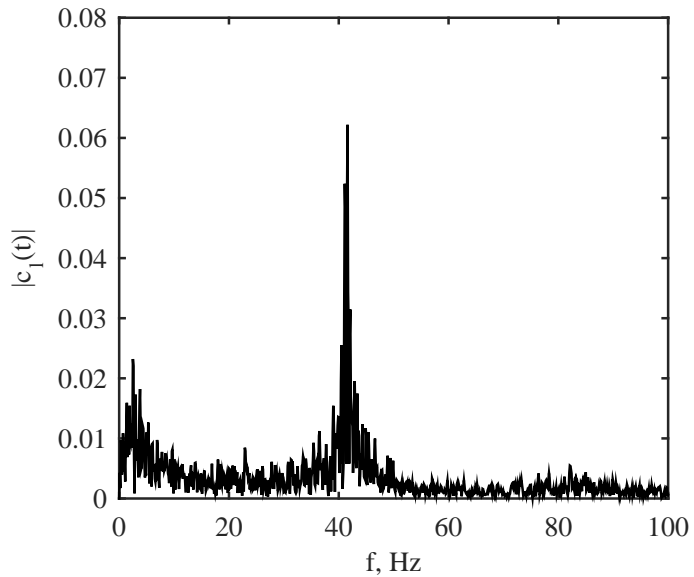

(a) Frequency content of $c_{l}(t)$ at $\alpha=150^{\circ}$.

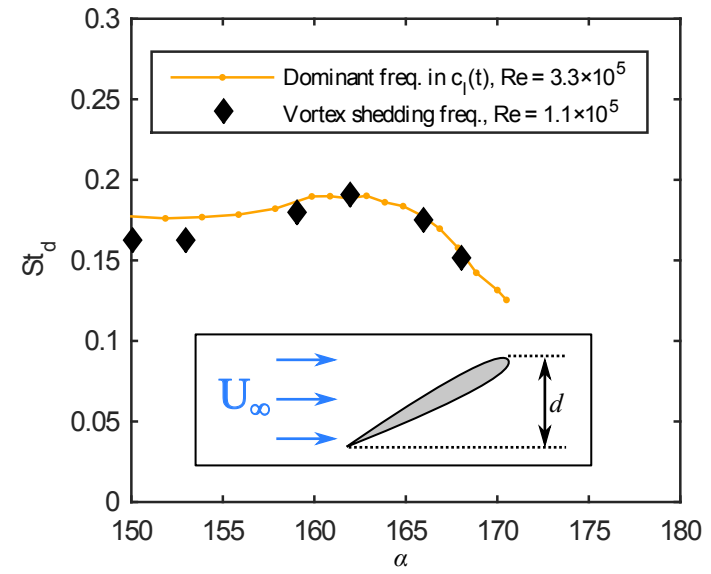

(b) Comparison of dominant frequency in unsteady lift with vortex shedding frequencies determined using flow field measurements [18].

Figure 8: Identification of vortex shedding frequencies (NACA 0012 at $R e=6.6 \times 10^{5}$ ).

\subsubsection{Unsteady Velocity Fields}

Time-resolved particle image velocimetry was used to measure the flow field over an uninstrumented NACA 0012 airfoil in forward and reverse flow in the UMD tunnel at $R e=$ $1.1 \times 10^{5}$ and $0^{\circ} \leq|\alpha| \leq 30^{\circ}$. Reference [15] describes the PIV setup in detail. For each angle of attack, 200 instantaneous velocity field measurements were collected using a 4 MPx Phantom v641 high-speed camera sampling the flow at $700 \mathrm{~Hz}$. The deep stall vortex shedding frequency under these conditions varied between $100 \leq f_{\mathrm{vs}} \leq 210 \mathrm{~Hz}$, depending on angle of attack. Unsteady velocity fields were calculated using the $2 \sigma$-variation of total velocity at each spatial location over the 200 measurements in order to highlight unsteady regions in the flow.

\section{Results and Discussion}

\subsection{Time-Averaged Airloads and Pressure Distributions}

The present section focuses on time-averaged airloads and pressure distributions; Section 4.2 will focus on unsteady airloads and pressure distributions. Figure 9 shows time- 
averaged airloads for a NACA 0012 airfoil for $0^{\circ} \leq \alpha \leq 180^{\circ}$ at $R e=3.3 \times 10^{5}$. Force balance measurements are compared with airloads calculated using the pressure integration technique outlined in Section 3.2.1. Similar to Figure 6(b), the plots in Figure 9 also show the unsteady airloads as a filled region surrounding the time-averaged airload curves. The time-averaged lift calculated through integration of the pressure distribution is in good agreement with force balance measurements for $20^{\circ} \leq \alpha \leq 160^{\circ}$. In forward flow $\left(0^{\circ} \leq \alpha \leq 20^{\circ}\right)$, the pressure gradients at the leading edge are high and the relatively sparse distribution of pressure sensors is unable to fully resolve the pre-stall suction and pressure peaks (Figure $4(\mathrm{a}))$ or the post-stall pressure side peak. Recall that geometric constraints imposed by the thin trailing edge prevented the installation of pressure sensors for $0.875 \leq x / c \leq 1$. In reverse flow $\left(160^{\circ} \leq \alpha \leq 180^{\circ}\right)$, the lack of physical pressure measurements restricts the resolution of suction and pressure expected here in reverse flow (Figure 4(d)). The consequences of these integration errors are observed in the lift and pitching moment curves. The discrepancies in the pitching moment curve are driven by the effect of integration error on the calculation of the moment arm between the quarter-chord and center of pressure. The moderate under-prediction of time-averaged lift and pitching moment from pressure integration likely results in a moderate under-prediction of the magnitude of the unsteady values as well. However, insight can still be gained from the following discussion since the trends of the time-averaged airloads calculated from pressure integration follow the force balance curves and show good agreement for high angles of attack where pressure gradients are low. It should also be noted that the agreement between force balance measurements and integrated pressure measurements is generally better for the three thick airfoils tested in the present work than for the NACA 0012 shown in Figure 9, due to less severe pressure gradients and a greater concentration of pressure measurements near the trailing edge.

Time-averaged pressure distributions for the four airfoils tested in the present work are shown in Figure 10 using contour plots. The uppermost contour plots show the pressure 

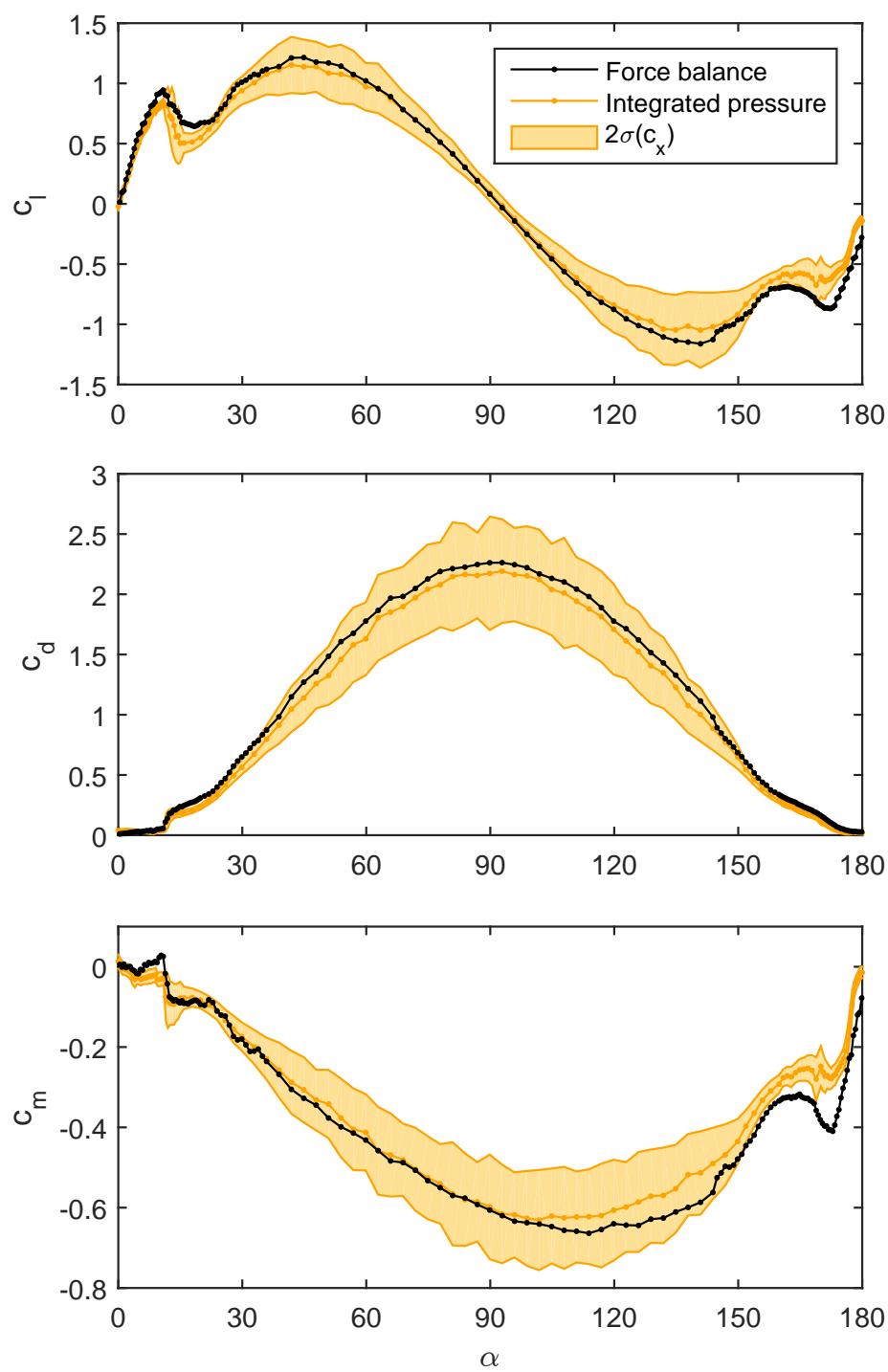

Figure 9: Time-averaged airloads of a NACA 0012 airfoil at $R e=3.3 \times 10^{5}$. 
distributions for the NACA 0012; the suction side is shown on the left (Figure 10(a)) while the pressure side is shown on the right (Figure 10(b)). In these contour plots, angle of attack is plotted along the abscissa with a minimum of $\alpha=0^{\circ}$ (pure forward flow) and a maximum of $\alpha=180^{\circ}$ (pure reverse flow). The chord position is plotted along the ordinate where $x / c=0$ is the geometric leading edge (blunt for the NACA 0012) and $x / c=1$ is the geometric trailing edge (sharp for the NACA 0012). The dotted lines on each plot mark the locations of pressure transducers. The sketches above the NACA 0012 contour plots illustrate the orientation of the airfoil at selected angles of attack, highlight the side of the airfoil shown in the contour plots, and show the definition of leading and trailing edge in the present work (fixed to the geometry of the airfoil). Note that for $90^{\circ}<\alpha<180^{\circ}$, the geometric trailing edge serves as the aerodynamic leading edge since the airfoil is operating in reverse flow. Refer to the sketch of the airfoil at $\alpha=150^{\circ}$.

Focusing on the suction side of the NACA 0012 airfoil (Figure 10(a), uppermost contour plot), for low angles of attack $\left(0^{\circ} \leq \alpha \leq 10^{\circ}\right)$ the pressure near the leading edge decreases with increasing angle of attack (shown in purple), consistent with a suction peak. In this angle of attack range, the stagnation point shifts along the pressure side of the airfoil away from the leading edge (Figure 10(b), upper plot). Discontinuities in the contours can be seen at $\alpha=12^{\circ}$ and $15^{\circ}$. These are consistent with the two-stage stall observed in Figure 9 . Turning attention back to the contour plot of the suction side of the NACA 0012, for $15^{\circ} \leq \alpha \leq 175^{\circ}$, the pressure distribution is nearly uniform since the flow is fully separated from the suction side of the airfoil here. However, on the pressure side of the airfoil, the pressure distribution is non-uniform and the center of pressure (dark red) shifts from near the leading edge towards the trailing edge with increasing angle of attack. These two trends are consistent with Pope's findings [11] and can also be observed in the "slices" of the contour plots given in Figures 4(b) and 4(c). The suction side maintains a relatively uniform distribution and the center of pressure on the pressure side shifts towards the trailing edge 

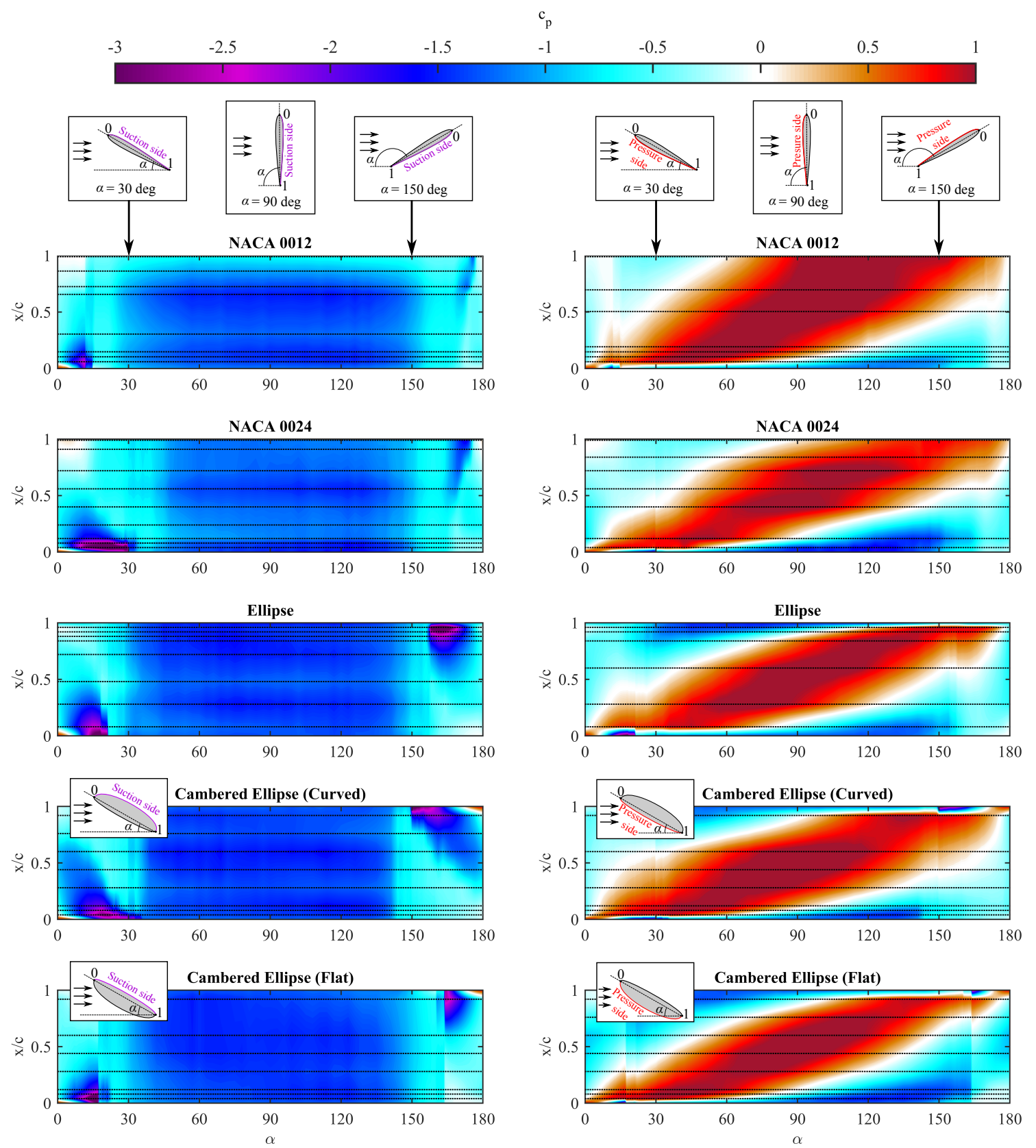

(a) Suction side.

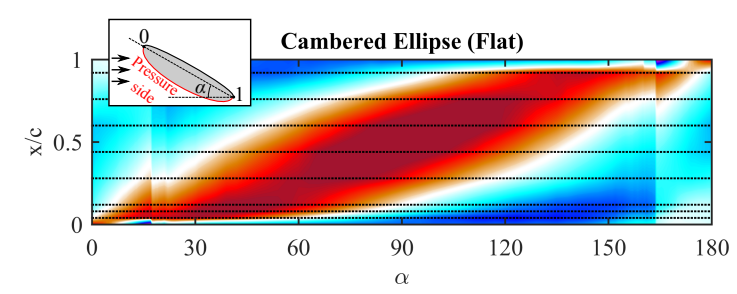

(b) Pressure side.

Figure 10: Time-averaged pressure distributions over four airfoils through $180^{\circ}$ angle of attack at $R e=6.6 \times 10^{5}$. Dashed lines indicate chordwise stations of pressure measurements. 
with increasing angle of attack. Figure 4(d) shows that the pressure decreases on the suction side of the airfoil in reverse flow $\left(\alpha=170^{\circ}\right)$; this is also observed in the contour plot for the NACA 0012 in Figure 10(a). In reverse flow, the NACA 0012 undergoes a thin-airfoil stall due to flow separation at the sharp aerodynamic leading edge [15]. As a result of this partially separated flow, the suction near the trailing edge in reverse flow $\left(170^{\circ} \leq \alpha \leq 180^{\circ}\right)$ is less than the suction near the leading edge in forward flow $\left(0^{\circ} \leq \alpha \leq 10^{\circ}\right)$.

The contour plots for the other airfoils share some similar features: a suction peak develops with increasing angle of attack in forward flow, the stagnation point moves aft of the leading edge, then stall occurs and the pressure distribution is nearly uniform on the suction side of the airfoil while the center of pressure on the pressure side moves towards the trailing edge. There are also several features of these contours that are unique to each airfoil. For example, the NACA 0024 airfoil has a much greater stall angle $\left(\alpha=29.5^{\circ}\right)$. Additionally, the NACA 0024 exhibits a trailing edge stall in forward flow. Examining the contour of the suction side (beginning at $\alpha=10^{\circ}$ ), it can be seen that the region of low pressure (blue) that originally extends from $x / c \approx 0.15$ to $x / c \approx 0.45$ affects a decreasing portion of the chord with increasing angle of attack. This indicates movement of the separation point towards the leading edge, consistent with a trailing edge stall [28].

Moving on to the elliptical airfoil, the pressure contours from $0^{\circ} \leq \alpha \leq 90^{\circ}$ are nearly mirror images of the contours from $90^{\circ} \leq \alpha \leq 180^{\circ}$ (mirrored about $x / c=0.5$ ). This symmetry stems from the fact that the airfoil is symmetric about both $x / c=0.5$ and $z / c=0$. The cambered elliptical airfoil contour plots are given twice: one set in which the curved surface acts as the suction side (i.e., the geometric upper surface as indicated in the sketch above the contour, and also in Figure 3), and another set in which the flatter surface acts as the suction side. Since the cambered elliptical airfoil is symmetric about $x / c=0.5$, each set of contour plots for the cambered elliptical airfoil are symmetric in the same way as the contour plots for the elliptical airfoil. When the curved surface acts as the suction side, the 
airfoil undergoes a trailing edge stall, similar to the NACA 0024 airfoil. Because the cambered elliptical airfoil is symmetric about the mid-chord, the same trailing edge stall that is observed in forward flow $\left(5^{\circ} \leq \alpha \leq 30^{\circ}\right)$ is seen also seen in reverse flow $\left(150^{\circ} \leq \alpha \leq 175^{\circ}\right)$. However, when the flatter surface of the cambered elliptical airfoil serves as the suction side, the airfoil undergoes a leading edge stall at $\alpha=17^{\circ}$ (represented by the discontinuity in the contour plot). This is likely due to a high adverse pressure gradient due to the curvature near the leading edge.

The three thick airfoils (NACA 0024, ellipse, and cambered ellipse) all exhibit aerodynamic hysteresis. The angle of attack at which the flow transitions between being attached over the suction side of the airfoil (at least partially) and being fully separated depends on the direction in which the angle of attack is changing. This phenomenon will be described in greater detail in Section 4.3, but it is important to note that the pressure data given in this section corresponds to data collected while the angle of attack was increased through stall events. For example, results for the cambered elliptical airfoil shown here are from measurements in which the angle of attack was changed from $0^{\circ}$ to $141^{\circ}$ and $180^{\circ}$ to $144^{\circ}$.

\subsection{Unsteady Airloads and Pressure Distributions}

The unsteady airloads and pressure distributions on the four airfoils are now considered. Recall that the filled region surrounding the time-averaged airload curves in Figures 6(b) and 9 represent the $2 \sigma$-variation of airloads acting on static airfoils and that the magnitude of the $2 \sigma$-variations is here referred to as the unsteady airloads. Figure 11 shows the unsteady lift (left column), drag (center column), and pitching moment (right column) for the four airfoils considered here (including the two orientations of the cambered elliptical airfoil). For the NACA 0012 and NACA 0024 airfoils, results are shown for both forward and reverse flow; recall that the reverse flow angle of attack is defined as $\alpha_{\mathrm{rev}} \equiv \alpha-180^{\circ}$. Only forward flow results are shown for the elliptical and cambered elliptical airfoil since these two airfoils are symmetric about $x / c=0.5$, giving similar behavior in forward and reverse flow. 

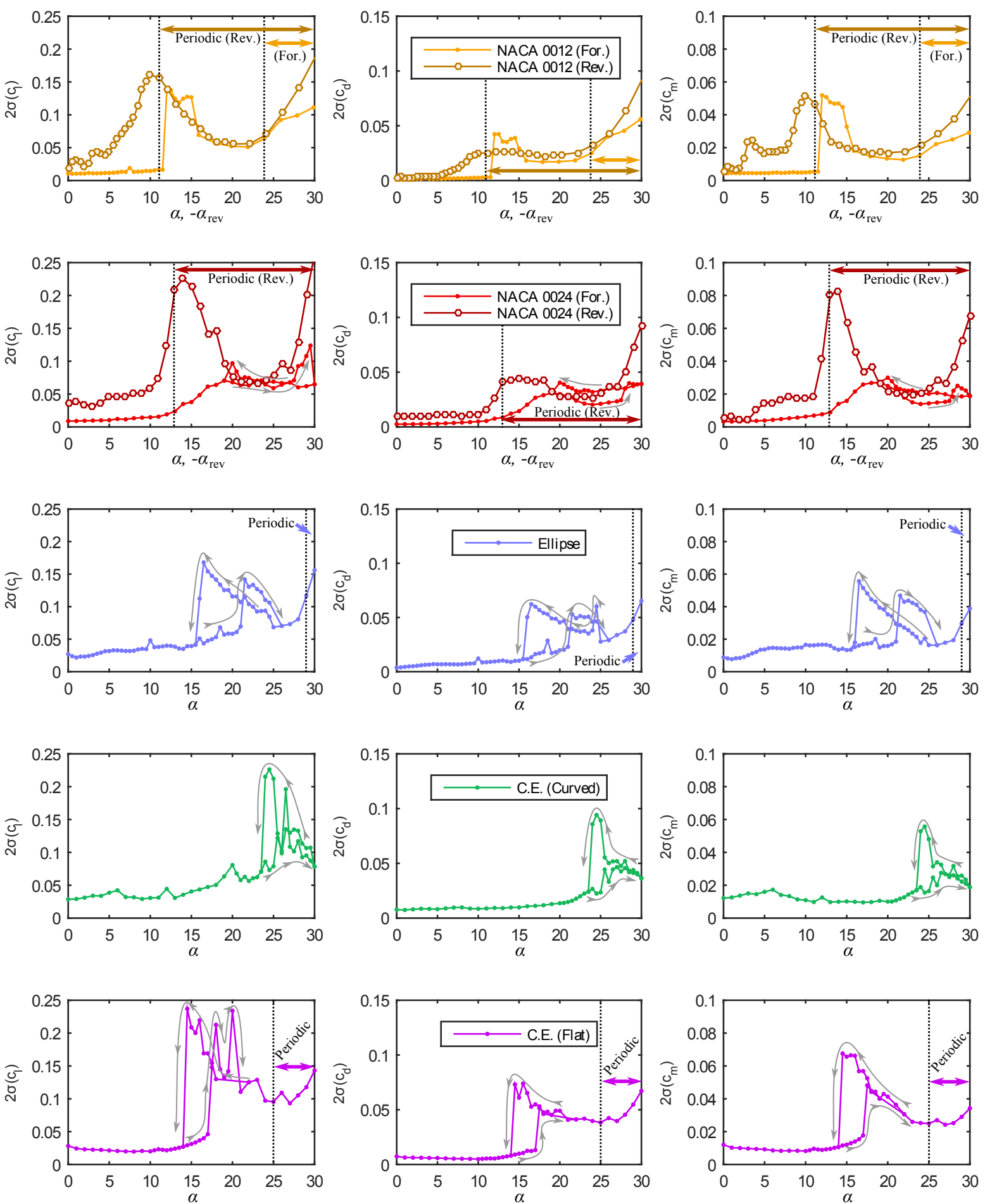

Figure 11: Unsteady airloads acting on four airfoils in forward and reverse flow at $R e=6.6 \times 10^{5}$. 
Throughout the following discussion on unsteady airloads, reference will also be made to Figure 12. The layout of these contours is identical to Figure 10, but Figure 12 shows unsteady pressure distributions $\left(2 \sigma\left(c_{p}\right)\right)$ rather than time-averaged. Unsteady pressure was previously shown as error bars in the pressure distribution of Figure 6(c). Reference will also be made to Figure 13 which shows unsteady total velocity fields along the suction side of the NACA 0012 airfoil at selected angles of attack in forward and reverse flow. Note that the velocity fields have been rotated to be fixed to the airfoil reference frame. The velocity field measurements used to calculate the unsteady flow fields were collected on an uninstrumented airfoil at $R e=1.1 \times 10^{5}[15]$. However, since most of the following discussion describes separated flows, the only significant effect of this lower Reynolds number is a slight decrease in stall angle.

Begin by examining the unsteady airloads acting on the NACA 0012 in forward flow (top row in Figure 11). The unsteady airloads are low in forward flow until the airfoil stalls at $\alpha=12^{\circ}$. The unsteady airloads generally remain at this elevated level for $12^{\circ} \leq \alpha \leq 15^{\circ}$ due to unsteady pressure fluctuations along the suction side of the airfoil. This is apparent in Figure 12(a) as a "band" of unsteady pressure (in blue, $12^{\circ} \leq \alpha \leq 15^{\circ}$ ). To understand this increase in unsteadiness more fully, the unsteady velocity fields are given in Figure 13(a). They show very low unsteadiness at $\alpha=2^{\circ}$ and $6^{\circ}$ because the flow is attached. However, once the airfoil is stalled $\left(\alpha=10^{\circ}\right.$ in Figure 13(a)), an unstable shear layer undulates near the suction side of the airfoil, leading to high unsteadiness along the entire chord. This is consistent with the band of unsteady pressure observed immediately after stall (Figure 12(a)).

Returning to the top row of Figure 11, the unsteady airloads in forward flow decrease in magnitude as the angle of attack is increased from $\alpha=15^{\circ}$ to $21^{\circ}$. The unsteady pressure distribution (Figure 12(a)) also decreases in this angle of attack range and is linked to the unsteady velocity field (Figure 13(a), $\alpha=21^{\circ}$ ). Here, the shear layer is stable (that is, 

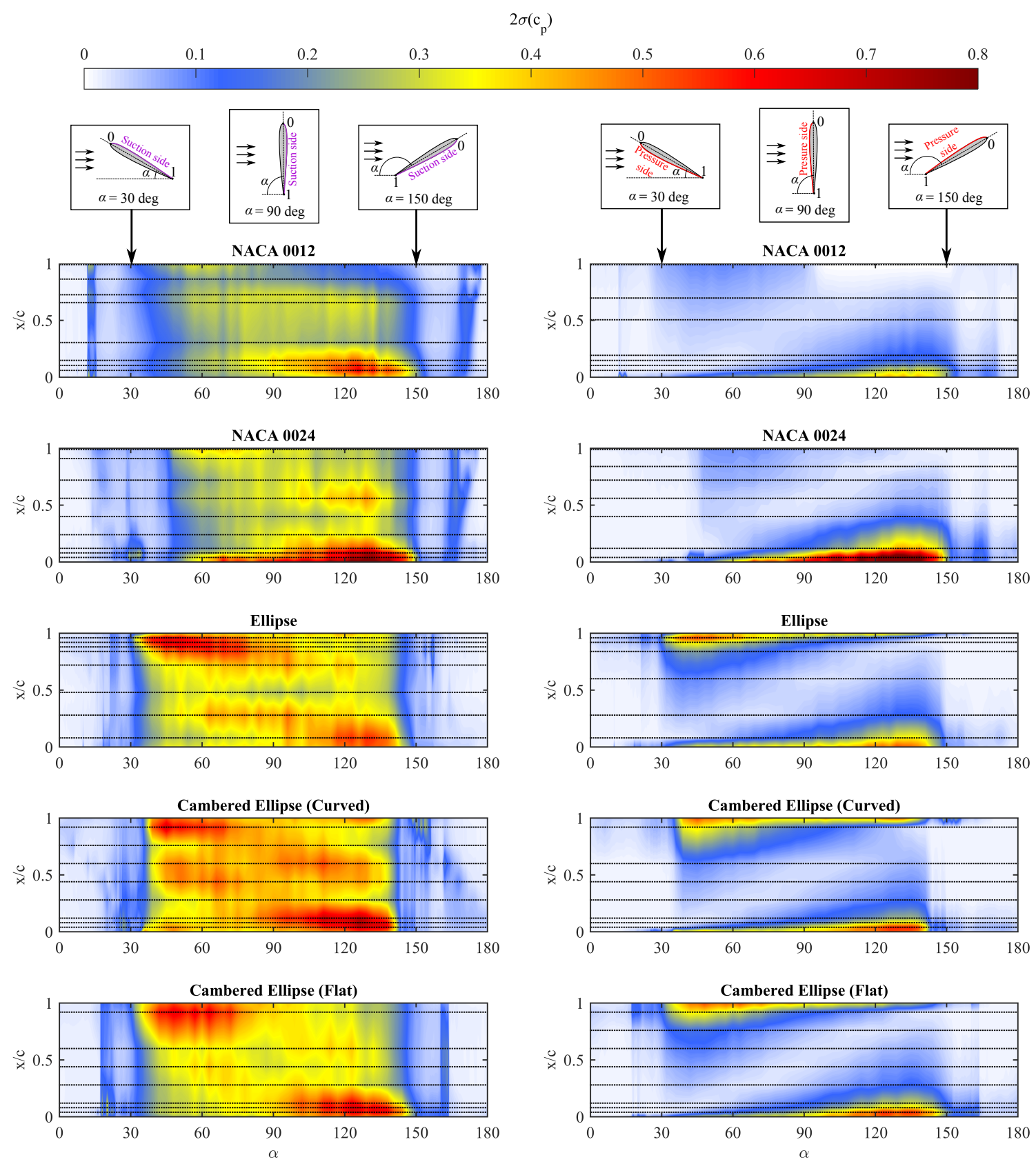

(a) Suction side.

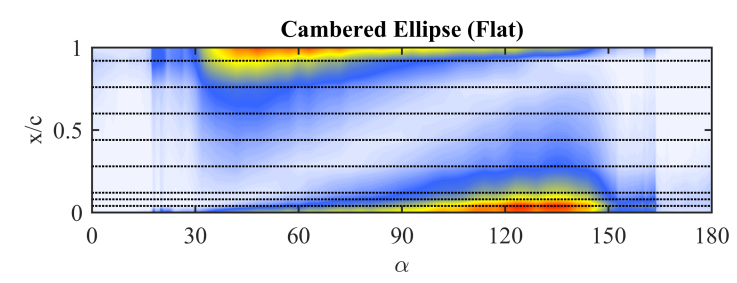

(b) Pressure side.

Figure 12: Distribution of unsteady pressure fluctuations for four airfoils through $180^{\text {circ }}$ angle of attack at $R e=6.6 \times 10^{5}$. Dashed lines indicate chordwise stations of unsteady pressure measurements. 
confined to a narrow region extending from the leading edge) and serves as a boundary between the external flow and a separated flow region near the airfoil. There is significantly less unsteadiness in the flow at the surface than at $\alpha=10^{\circ}$. The unsteadiness near the surface increases as the angle of attack is increased to $\alpha=30^{\circ}$, consistent with the increase in unsteady airloads observed in Figure 11. It should be noted that the airloads become periodic for $\alpha \geq 24^{\circ}$; a distinct vortex shedding frequency was detected using the approach described in Section 3.2.4. The measured vortex shedding frequency will be described later in this section.

Next, consider the unsteady airloads on the NACA 0012 in reverse flow. As the reverse flow angle of attack is increased, the unsteady airloads increase more quickly than in forward flow. In reverse flow, flow separation occurs at the blunt aerodynamic trailing edge as well as at the sharp aerodynamic leading edge. This leads to moderate unsteadiness in the flow field at angles of attack as low as $-\alpha_{\text {rev }}=3^{\circ}$ (Figure 13(b)). As the angle of attack is increased to $-\alpha_{\text {rev }}=6^{\circ}$, flow separation at the sharp geometric trailing edge forms an unstable shear layer with the greatest unsteadiness over $1 \geq x / c \geq 0.4$. At $-\alpha_{\text {rev }}=9^{\circ}$, the high unsteadiness extends over the entire chord. This expansion of the region of unsteadiness in the chordwise direction is also reflected in the unsteady pressure distribution (blue band in the upper plot of Figure $12(\mathrm{a}), 180^{\circ} \geq \alpha \geq 170^{\circ}$, interpreted from right to left). This angle of attack range corresponds with the turbulent reverse flow wake regime; the wake has no periodic flow features [18]. Returning to Figure 12, note that the reverse flow airloads become periodic at $-\alpha_{\mathrm{rev}}=11^{\circ}$; this marks the beginning of the deep stall vortex shedding regime [18]. Like in forward flow, the unsteady airloads then begin to decrease (up to $-\alpha_{\text {rev }}=22^{\circ}$ ) as a stable shear layer forms a boundary with the separated flow region $\left(\right.$ see $-\alpha_{\text {rev }}=21^{\circ}$ in Figure 13(b)). For $-\alpha_{\text {rev }}>22^{\circ}$, the unsteady airloads in reverse flow increase, exceeding the corresponding unsteady airloads in forward flow. Indeed, the flow unsteadiness at the surface of the airfoil at $|\alpha|=30^{\circ}$ is higher in reverse flow than in forward flow (Figure 13). 
$2 \sigma\left(|\mathrm{U}| / \mathrm{U}_{\infty}\right)$
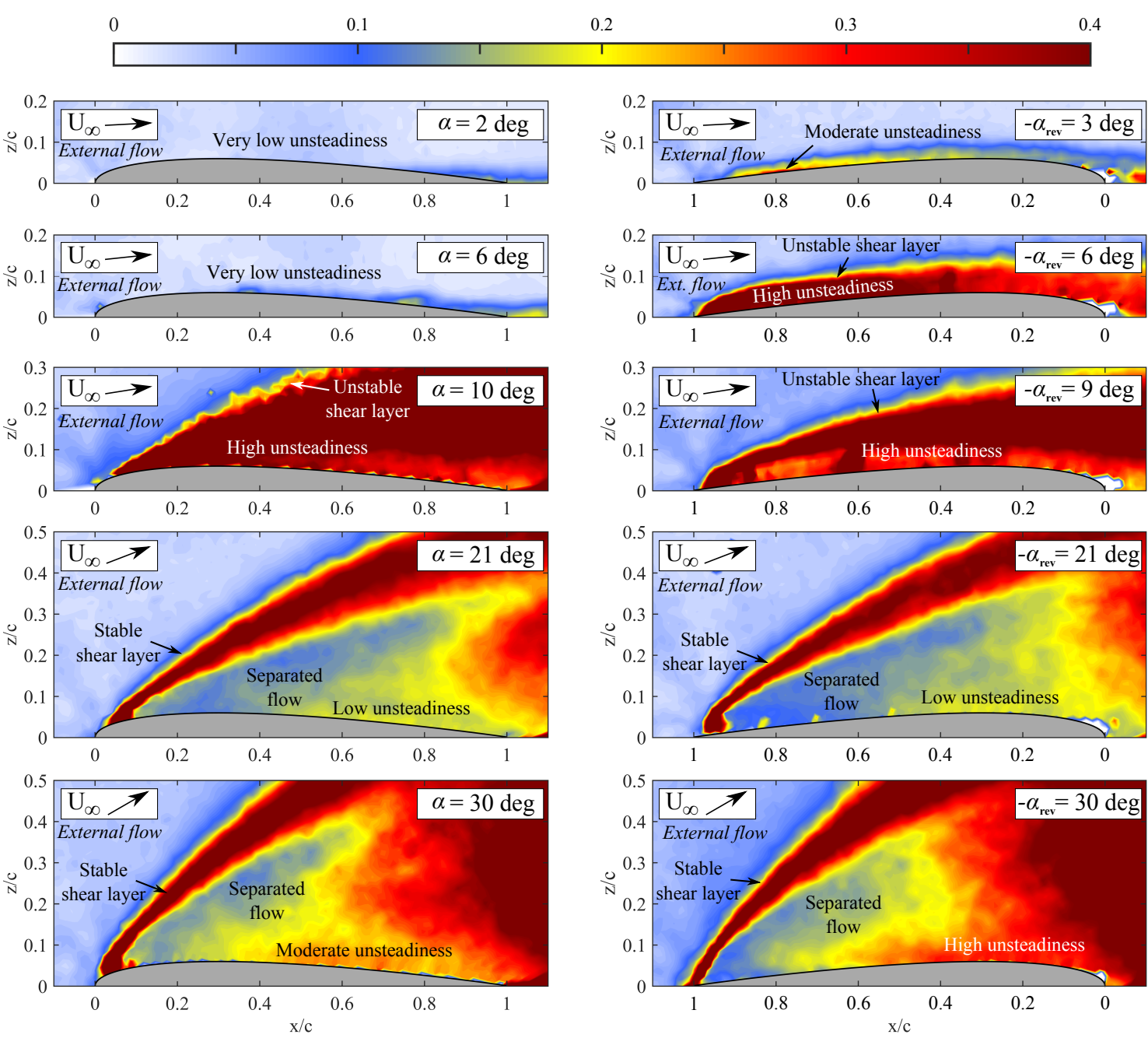

(a) Forward Flow.

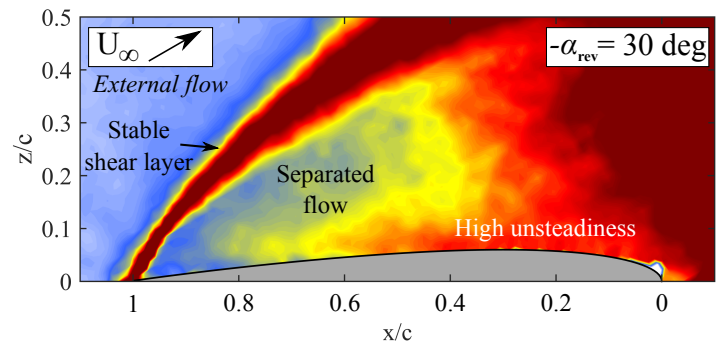

(b) Reverse Flow.

Figure 13: Unsteady velocity fields near the suction side of a NACA 0012 at $R e=1.1 \times 10^{5}$.

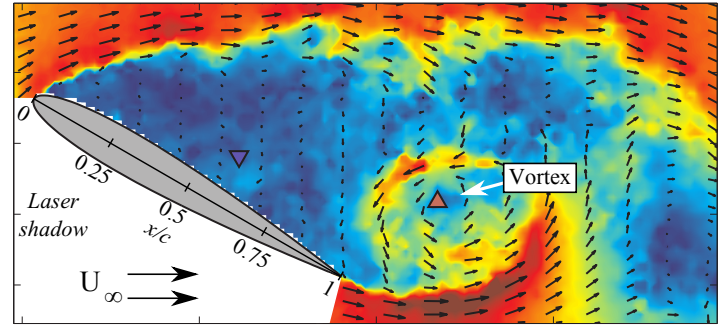

(a) Forward flow, $\alpha=30^{\circ}$.

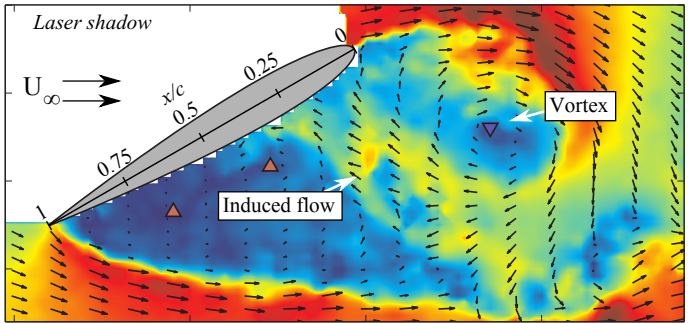

(b) Reverse flow, $-\alpha_{\text {rev }}=30^{\circ}[18]$.

Figure 14: Instantaneous flow field measurement of the suction side of a NACA 0012 airfoil at $R e=1.1 \times 10^{5}$. 
This is because the deep stall vortex shedding characteristics differ in forward and reverse flow. Figure 14 shows instantaneous velocity field measurements in forward and reverse flow at similar stages of vortex development. In both cases, a vortex periodically forms and sheds from the aerodynamic trailing edge. The vortex generally forms closer to the airfoil in reverse flow (Figure 14(b)), inducing flow near the geometric leading edge (aerodynamic trailing edge). Note that the forward flow instantaneous measurement (Figure 14(a)) shows that there is no induced flow near the surface of the airfoil. The time-series of the instantaneous measurements (not shown) confirms that little flow is induced by vortex shedding near the surface of the airfoil in forward flow at this angle of attack. This contrasts with reverse flow, where the unsteadiness in the induced flow is responsible for the high unsteady pressure near the blunt geometric leading edge. Vortices also form in the shear layer extending from the sharp geometric trailing edge (aerodynamic leading edge), but they are positioned further downstream of the airfoil and do not affect the flow at the surface as much as the vortices shedding from the blunt leading edge [18].

The behavior of the NACA 0024 in reverse flow (second row of Figure 11) is qualitatively similar to the NACA 0012 in reverse flow. The unsteady airloads on the NACA 0024 increase with reverse flow angle of attack, although the rate of increase is less than the NACA 0012, likely due to the greater airfoil thickness. The unsteady airloads then become periodic and begin to decrease as the shear layer becomes stable and moves away from the suction side. The unsteady airloads increase again as the vortex shedding becomes stronger. The behavior of the NACA 0024 in forward flow is dramatically different from the NACA 0012. In Section 4.1, the trailing edge stall characteristics of the NACA 0024 were described. This manifests as a more gradual increase in the unsteady airloads with increasing angle of attack (rather than the rapid increase in unsteady airloads observed for the NACA 0012 during its leading edge stall in forward flow). Note that for the NACA 0024 in forward flow, deep stall occurs at $\alpha=30^{\circ}$ and the airloads become periodic (i.e., vortex shedding begins) at 
$\alpha=42^{\circ}$; these features are not on the scale of Figure 11.

Attention is now turned to the airfoils featuring a blunt geometric trailing edge. The unsteady airloads on the elliptical airfoil are shown in the third row of Figure 11. As was mentioned previously, the thick airfoils undergo aerodynamic hysteresis. The grey arrows indicate the direction in which angle of attack is changed. For now, consider increasing angle of attack only. Beginning at low angles of attack $\left(0^{\circ} \leq \alpha \leq 10^{\circ}\right)$, it can be seen that the unsteady airloads of the elliptical airfoil are greater than either of the two NACA-series airfoils in forward flow (due to flow separation at the blunt trailing edge), but are generally less than the unsteady airloads of the NACA-series airfoils in reverse flow. Note, however, that for $0^{\circ} \leq \alpha \leq 10^{\circ}$ the unsteady airloads on the elliptical airfoil and the NACA 0024 in reverse flow are nearly the same since both have a thick blunt aerodynamic trailing edge here. Unlike the NACA 0024, the unsteady airloads on the elliptical airfoil remain low with increasing angle of attack since the flow remains mostly attached until full flow separation (and a corresponding rapid increase in unsteady airloads) occurs at $\alpha=21^{\circ}$. Similar to the NACA-series airfoils, the unsteady airloads decrease as the angle of attack is increased post-stall $\left(21^{\circ} \leq \alpha \leq 26^{\circ}\right)$ and the leading edge shear layer moves away from the airfoil. As the angle of attack is increased further $\left(\alpha \geq 26^{\circ}\right)$, the unsteady airloads increase (similar to the NACA-series airfoils) and eventually become periodic.

The unsteady airloads on the cambered elliptical airfoil depend on which surface of the airfoil serves as the suction side. The fourth row of Figure 11 shows the unsteady airloads for the curved surface acting as the suction side. Here, unsteady airloads generally gradually increase with angle of attack. Since the airfoil exhibits a trailing edge stall in this orientation, there is not as large of a rapid increase of unsteady airloads as the airfoil increases in angle of attack through stall. The fifth row of Figure 11 shows the unsteady airloads for when the flatter surface acts as the suction side. Here, the airfoil undergoes a leading edge stall, so the unsteady airloads increase rapidly (similar to the NACA 0012 and elliptical airfoil). 
Figure 12 shows a corresponding rapid increase in the unsteady pressure along the suction side at $\alpha=17.5^{\circ}$. Like the NACA series airfoils and elliptical airfoil, the unsteady airloads (and unsteady pressure distribution) generally decrease in magnitude post-stall and then begin to increase at the onset of vortex shedding. There is an important difference, however, between the NACA series airfoils and the airfoils with a blunt geometric trailing edge. In reverse flow, the unsteady airloads of the NACA series airfoils become periodic at a relatively low angle of attack $\left(-\alpha_{\text {rev }} \approx 12^{\circ}\right)$, whereas airloads become periodic at much greater angles of attack $\left(\alpha \geq 25^{\circ}\right)$ for airfoils with a blunt trailing edge. This is worth noting because the periodicity of the airloads could induce rotor blade vibrations if the vortex shedding frequency is close a natural structural frequency.

Figure 15 expands on Figure 11 by showing the unsteady airloads and dimensionless vortex shedding frequency, $S t_{d}$, for the four airfoils for $0^{\circ} \leq \alpha \leq 180^{\circ}$. This figure segments the results into four angle of attack ranges: low- $\alpha$ forward flow $\left(0^{\circ} \leq \alpha \leq 30^{\circ}\right.$, attached flow and through stall), high- $\alpha$ forward flow $\left(30^{\circ} \leq \alpha \leq 90^{\circ}\right.$, deep stall), high- $\alpha$ reverse flow $\left(90^{\circ} \leq \alpha \leq 150^{\circ}\right.$, deep stall), and low- $\alpha$ reverse flow $\left(150^{\circ} \leq \alpha \leq 180^{\circ}\right.$, deep stall to nearly fully attached flow). For all airfoils, there is a local maximum in the unsteady lift in the high- $\alpha$ forward flow range. The unsteady lift has a local minimum near $\alpha=90^{\circ}$ where the unsteady drag reaches its maximum since the airfoil is perpendicular to the freestream. The unsteady pitching moment generally increases rapidly at the beginning of the high- $\alpha$ forward flow range, then slowly increases through most of the high- $\alpha$ reverse flow range before decreasing rapidly (as the unsteady lift and drag decrease).

There is another important difference in the behavior of the unsteady airloads acting on the two NACA series airfoils as compared to the elliptical airfoils. The unsteady lift and drag of the elliptical and cambered elliptical airfoils are generally symmetric about $\alpha=90^{\circ}$; recall that symmetry was seen previously for these airfoils in the time-averaged pressure distributions (Figure 10). The unsteady lift and drag of the NACA series airfoils is generally 

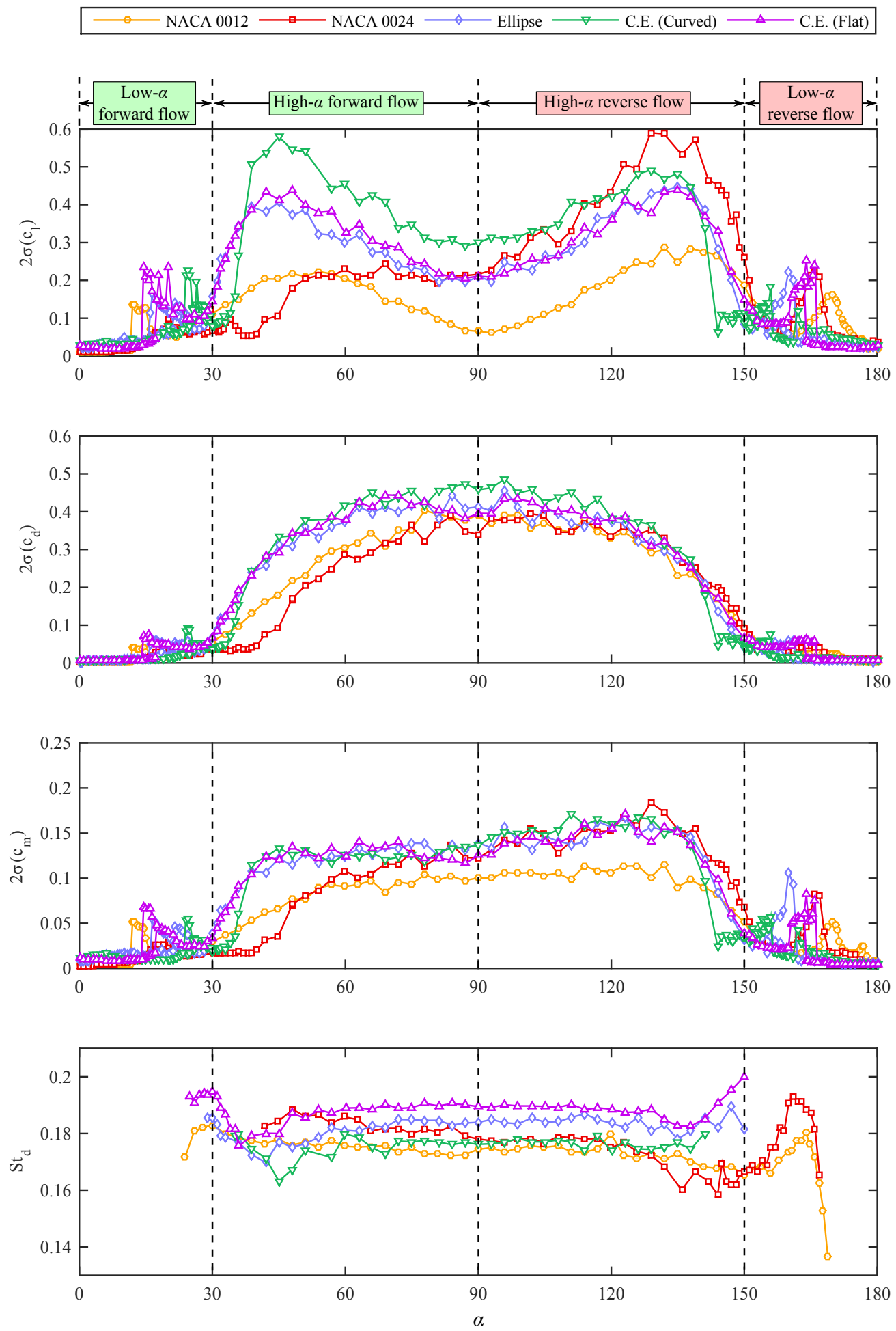

Figure 15: Magnitude and frequency of unsteady airloads acting on four airfoils through $180^{\circ}$ at $R e=$ $6.6 \times 10^{5}$. 
greater in the high- $\alpha$ reverse flow range than in the high- $\alpha$ forward flow range. This can be linked to the unsteady pressure distributions in Figure 12. For the NACA series airfoils, the unsteady pressures are greatest near the blunt aerodynamic trailing edge $(x / c=0)$ in the high- $\alpha$ reverse flow range (suction side and pressure side). Recall that vortex shedding in reverse flow leads to the unsteady induced flow near the blunt aerodynamic trailing edge. In the high- $\alpha$ forward flow range, it is believed that vortex formation occurs further downstream in the wake, causing the unsteady airloads to be less than those found in the high- $\alpha$ reverse flow range.

To summarize, the dominant source of unsteady airloads in the high- $\alpha$ range (forward and reverse) is induced flow from bluff body vortices that form as the flow turns around a blunt aerodynamic trailing edge. For the elliptical and cambered elliptical airfoils, this occurs in both forward and reverse flow, leading to symmetry of the unsteady pressure contours of Figure 12. For the NACA series airfoils, this phenomenon only occurs in the high- $\alpha$ reverse flow range since this is where the blunt geometric leading edge serves as the aerodynamic trailing edge.

The vortex shedding frequency from the four airfoils is now discussed in greater detail (lower plot of Figure 15). Recall that the dimensionless vortex shedding frequency used in the present work is $S t_{d}=f d / U_{\infty}$, where $d$ is the projected diameter of the airfoil at a given angle of attack (see inset of Figure 8(b)). Also recall that the vortex shedding frequency was identified by examining the frequency content of $c_{l}(t)$ (Section 3.2.4). At the beginning of the high- $\alpha$ forward flow range, the vortex shedding frequency of nearly all airfoils decreases slightly, but then remain generally constant until the end of the high$\alpha$ reverse flow range when the shedding frequency increases slightly. Vortex shedding for the NACA series airfoils continues into the low- $\alpha$ reverse flow range with the appearance of "hooks" in the plot. At low angles of attack, the leading edge shear layer is in close proximity to the trailing edge shear layer, causing shear layer interactions that affect the 
vortex shedding frequency here [18].

The vortex shedding frequency at high angles of attack also depends on the shape of the surface of the airfoil oriented upstream as this affects the angle at which the shear layers depart from the airfoil (i.e., separation angles) $[29,30,31,18]$. More streamlined bodies have lower separation angles, and thus greater vortex shedding frequencies. Focusing on $\alpha=90^{\circ}$, the cambered elliptical airfoil with the flat surface acting as the suction side has the greatest vortex shedding frequency $\left(S t_{d}=0.189\right)$. This is because the curved surface faces upstream in this orientation. When oriented perpendicular to the flow, this curved surface is the most streamlined of the airfoils tested, giving the greatest vortex shedding frequency. The next most streamlined surface is the elliptical airfoil, and hence it has a slightly lower vortex shedding frequency $\left(S t_{d}=0.184\right)$. The NACA series airfoils and the flat surface of the cambered elliptical airfoil are least streamlined in this orientation, causing these airfoils to have the lowest shedding frequencies $\left(0.174 \leq S t_{d} \leq 0.178\right)$. Finally, note that the vortex shedding frequencies of the NACA series airfoils decrease slightly as the angle of attack passes through the high- $\alpha$ forward and reverse flow ranges $\left(30^{\circ} \leq \alpha \leq 150^{\circ}\right)$. This is likely due to changes in the separation angles of the shear layers; in the high- $\alpha$ forward flow range, the blunt edge serves as the aerodynamic leading edge whereas the sharp edge serves as the aerodynamic leading edge in the high- $\alpha$ reverse flow range.

\subsection{Aerodynamic Hysteresis}

Aerodynamic hysteresis occurs when flow reattachment from fully stalled conditions takes place at a lower angle of attack than static stall. The direction in which the static angle of attack changes affects the angle at which the airfoil transitions between being stalled and unstalled. This phenomenon has been well documented and typically affects thick airfoils with a rounded leading edge $[32,33]$. The effect of aerodynamic hysteresis on the time-averaged airloads and flowfields for the airfoils tested in the present work are given in References [15, 9]. 


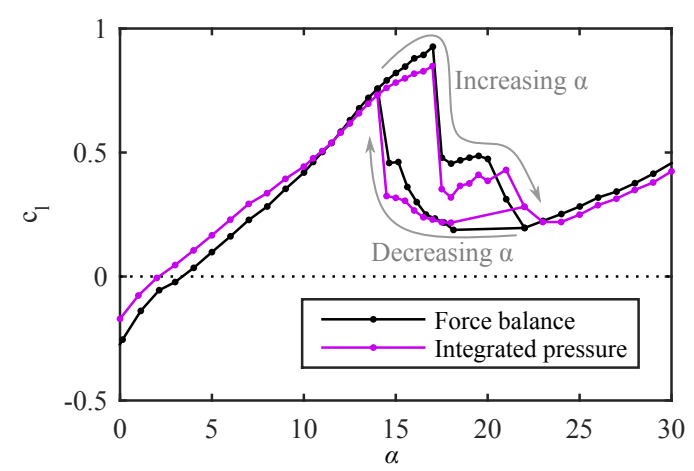

(a) Time-averaged lift curve.
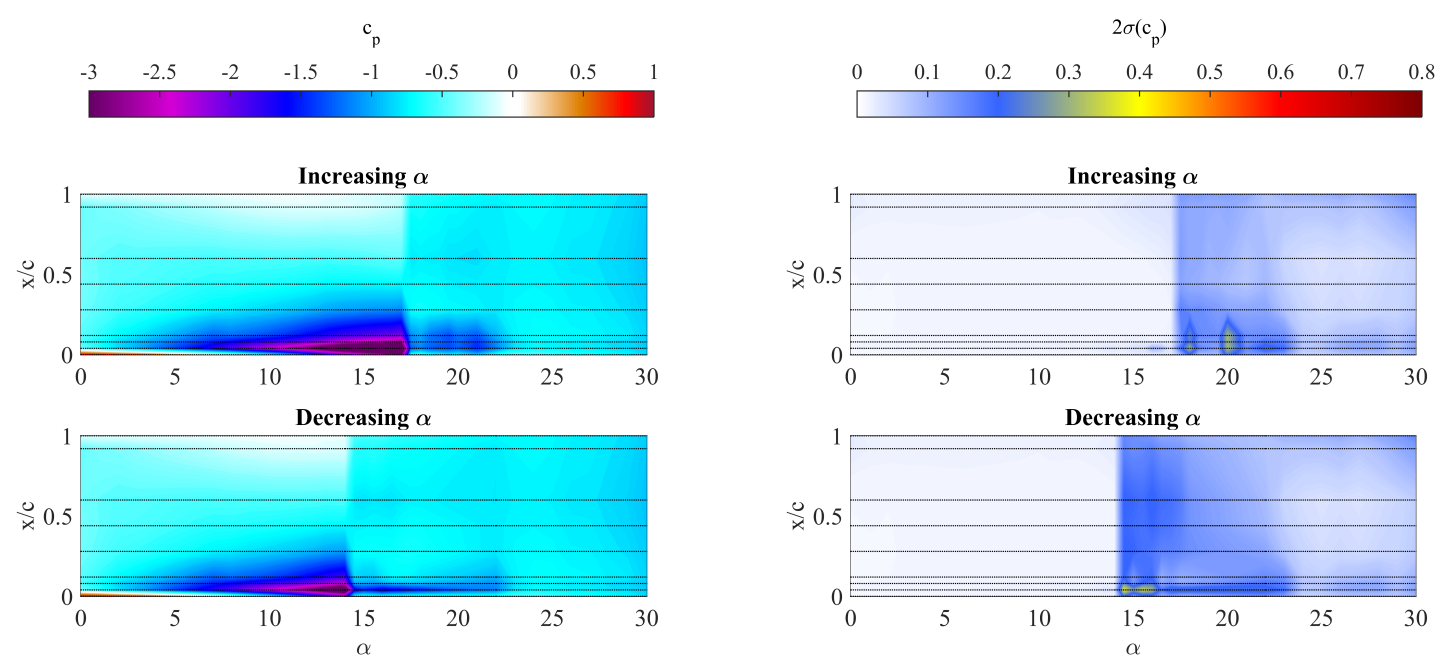

(b) Time-averaged pressure distribution (suction (c) Unsteady pressure distribution (suction side). side).

Figure 16: Effect of aerodynamic hysteresis on the airloads and pressure distributions of with the flatter side of the cambered elliptical airfoil serving as the suction side at $R e=6.6 \times 10^{5}$. 
Figure 16 shows the effect of aerodynamic hysteresis on the cambered elliptical airfoil with the flatter side serving as the suction side. Figure 16(a) shows the time-averaged lift curve measured using a force balance and calculated using pressure integration. Following the increasing- $\alpha$ branch, there is a two-stage static stall at $\alpha=17^{\circ}$ and $20^{\circ}$. Now following the decreasing- $\alpha$ branch, it can be seen the airfoil becomes unstalled at $\alpha=14^{\circ}, 3^{\circ}$ less than the onset of the first stage of static stall.

Figure 16(b) shows the time-averaged pressure distributions on the suction side for increasing and decreasing angles of attack; Figure 16(c) shows corresponding unsteady pressure distributions. Focusing first on the increasing angle of attack contours, the time-averaged contour shows a region of low pressure near the leading edge that grows with angle of attack while the unsteady pressure distribution is nearly uniformly zero since the flow is mostly attached. At the onset of the fist stage of stall, the region of time-averaged suction near the leading edge decreases in magnitude while there is an abrupt increase in the unsteady pressure distribution. At $\alpha=18^{\circ}$ and $20.5^{\circ}$, there are pockets of high unsteady pressure fluctuations near the leading edge. These manifest as peaks in the unsteady airloads corresponding to the cambered elliptical airfoil in Figure 11 and are likely due to unsteadiness in the boundary layer separation point. As the angle of attack is increased beyond the second stage of the stall $\left(\alpha>23^{\circ}\right)$, the unsteady pressure distribution generally decreases in magnitude as the leading edge boundary layer moves further from the suction side of the airfoil.

Next, consider the pressure distributions when the angle of attack is decreasing. The time-averaged pressure distribution shows that the suction near the leading edge resumes at $\alpha=14^{\circ}$, consistent with the force measurements given in Figure 16(a). It is important to note that the unsteady pressure distribution is greatest in magnitude for $14^{\circ}<\alpha \leq 17.5^{\circ}$ as the angle of attack approaches reattachment. Similar to the increasing angle of attack unsteady pressure distribution, pockets of high unsteadiness are located near the leading 
edge.

Aerodynamic hysteresis has a detrimental effect on both the time-averaged and unsteady airloads acting on an airfoil. Referring back to the unsteady airloads shown in Figure 11, airfoils which exhibit aerodynamic hysteresis have greater unsteady airloads as angle of attack is decreased through stall. This is attributed to unsteadiness in the leading edge shear layer coming into close proximity with the suction side of the airfoil before flow reattachment occurs.

\subsection{Reynolds Number Effects}

Rotor blades on wind turbines and helicopters encounter a wide range of Reynolds numbers due to the spanwise veolocity gradient that arises from rotational motion. For example, the Reynolds number range in the reverse flow region of a high-speed helicopter is $0 \leq R e \leq 1.5 \times 10^{6}[4,9]$. Therefore, it is important to understand the effect of Reynolds number on unsteady airloads. Figure 17 shows the unsteady airloads for two representative airfoils: a NACA 0012 (in reverse flow) and the cambered elliptical airfoil with the flatter side acting as the suction side (symmetric airloads in forward and reverse flow). Before examining the features of the unsteady airload curves of these airfoils, it is important to note the measurement noise. Recall that the unsteady airloads are derived quantities from time-resolved pressure measurements. Each pressure transducer had some electrical noise associated with it, thus the integrated time-resolved airloads also contained noise. Table 1 lists the maximum noise of each airload at each Reynolds number tested. The noise was calculated by integrating the unsteady airloads for $5 \mathrm{~s}$ while the wind tunnel was off. Since the unsteady airloads are non-dimensionalized by dynamic pressure, the signal-to-noise ratio in the unsteady airloads increases with increasing Reynolds number.

Referring to Figure 17(a), it can be seen that the unsteady airloads acting on the NACA 0012 in reverse flow are generally insensitive to Reynolds number. The sharp leading edge forces flow separation, meaning that the separation point (and thus the unsteady 

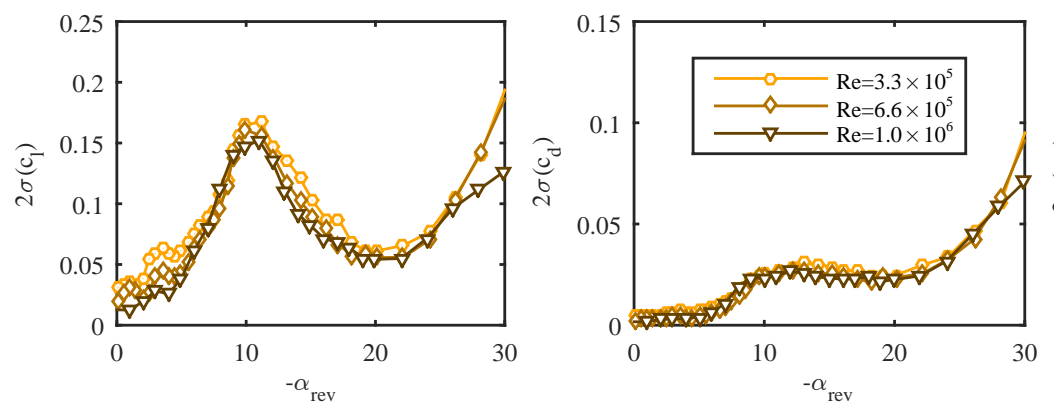

(a) NACA 0012
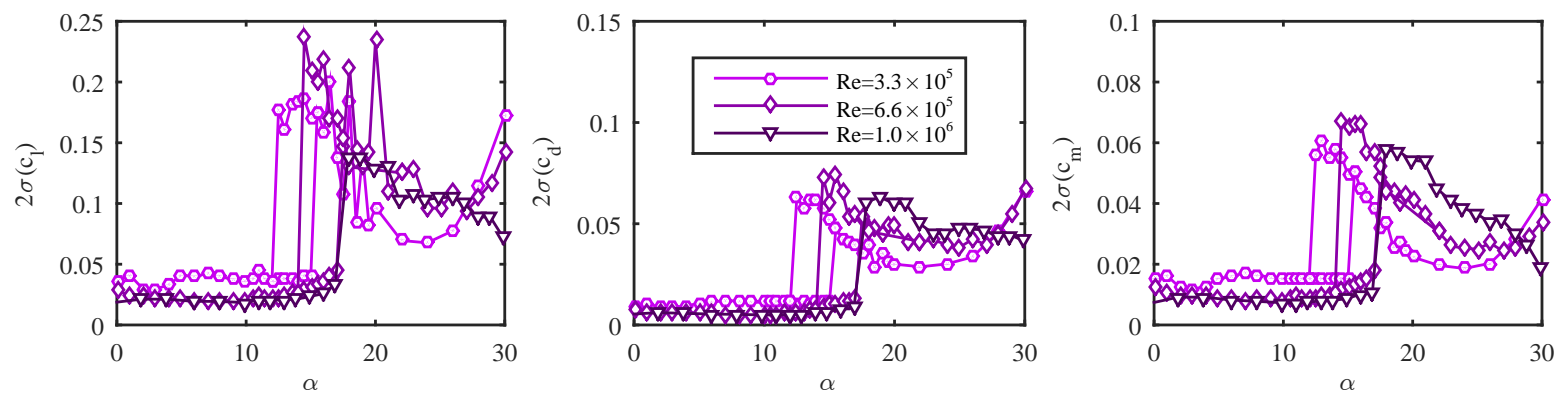

(b) Cambered ellipse (flat).

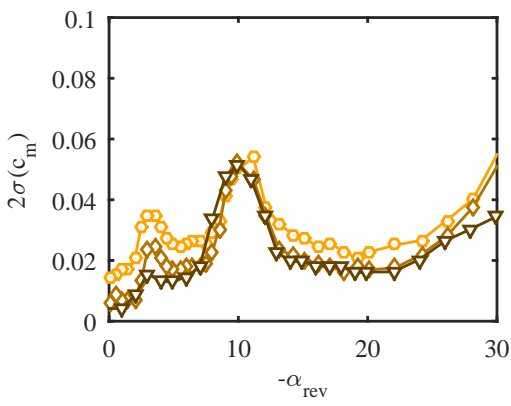

$$
\text { , }
$$


$\left(0^{\circ} \leq \alpha \leq 12^{\circ}\right)$, the unsteady airloads are greatest for $R e=3.3 \times 10^{5}$, although the signalto-noise ratio is low in this angle of attack range for this Reynolds number. The stall angle is increased with increasing Reynolds number leading to a shift in the rapid increase of unsteady airloads. The maximum unsteady airloads are nearly the same for all three Reynolds numbers, although there are no lift "spikes" observed for in the unsteady lift curve at $R e=1.0 \times 10^{6}$. In the post-stall region, the unsteady drag generally increases with Reynolds number, leading to greater pitching moment.

In summary, the unsteady airloads on a NACA 0012 airfoil are generally insensitive to Reynolds number in reverse flow since the flow separation point is fixed at the sharp aerodynamic leading edge. The unsteady airloads of the NACA 0012 in forward flow, as well as the NACA 0024 in both forward and reverse flow, are mildly sensitive to Reynolds number (not shown) since flow transition and separation points vary with Reynolds number. The cambered elliptical airfoil with the flatter side serving as the suction side shows some sensitivity to Reynolds number, specifically in delaying of stall and the post-stall unsteady airloads. These findings hold true for elliptical airfoil and the cambered elliptical airfoil with the curved side acting as the suction side (not shown).

\section{Conclusions}

Time-averaged and unsteady airloads and pressure distributions were calculated from time-resolved pressure measurements integrated using a panel-style pressure integration scheme. This was done for four airfoils at high angles of attack and in reverse flow $\left(0^{\circ} \leq \alpha \leq 180^{\circ}\right)$ Comparisons were made with unsteady velocity field measurements to determine the source of unsteady airloads. Five conclusions can be drawn from the analysis presented here.

1. Unsteady airloads in forward and reverse flow $\left(0^{\circ} \leq|\alpha| \leq 30^{\circ}\right)$. Unsteady airloads are closely linked to the type of static stall exhibited by an airfoil. Airfoils that exhibit a leading edge stall (e.g., NACA 0012 in forward flow, elliptical airfoil, and cambered 
elliptical airfoil with the flat surface acting as the suction side) have a rapid increase in unsteady airloads at the onset of stall. Airfoils that exhibit a trailing edge stall (e.g., NACA 0024 in forward flow and cambered elliptical airfoil with the curved surface acting as the suction side) have unsteady airloads that increase gradually. Airfoils that exhibit a thin-airfoil stall (e.g., NACA series airfoils in reverse flow) have unsteady airloads that increase at a moderate rate due progressively increasing amounts of flow separation originating at the sharp aerodynamic leading edge. In all cases, the unsteady airloads are greatest when the shear layer is unstable and near suction side of the airfoil. This is a result of the turbulent wake regime observed in prior work [18].

2. Unsteady airloads at high angles of attack $\left(30^{\circ} \leq \alpha \leq 150^{\circ}\right)$. This angle of attack range corresponds with the deep stall vortex shedding regime. Here, unsteady airloads are greatest near a blunt aerodynamic trailing edge since the shear layer can curl around, forming a vortex that induces unsteady flow near surface on suction side. It is believed that the lower unsteady airloads observed for airfoils with a sharp aerodynamic trailing edge results from vortex shedding occurring further downstream.

3. Vortex shedding frequency. For each airfoil tested, the Strouhal number (based on the projected diameter of the airfoil) is nearly constant for $30^{\circ} \leq \alpha \leq 150^{\circ}$. The value depends on the shape of the upstream surface; airfoils with a more streamlined upstream surface have a greater vortex shedding frequency, consistent with previous findings [18].

4. Aerodynamic hysteresis. In the angle of attack range $0^{\circ} \leq \alpha \leq 30^{\circ}$, unsteady airloads acting on thick airfoils are greatest when the angle of attack is being decreased through stall. This is a result of an unstable shear layer approaching the suction side of the airfoil. 
5. Reynolds number effects. For airfoils exhibiting a leading edge stall or trailing edge stall, the stall angle (and corresponding onset of high unsteady airloads) increases with angle of attack. Airfoils that undergo a thin-airfoil-like stall have unsteady airloads that are independent of Reynolds number due to the fixed separation point at the sharp aerodynamic leading edge.

The results of the present work provide insight on the unsteady airloads acting on static airfoils at angles of attack through $180^{\circ}$. This represents a fundamental model of flow around starting/stopped wind turbine rotor blades and in the vicinity of the reverse flow region of a high-speed helicopter where rapid changes in the local freestream lead to a wide range of angles of attack. Airfoils with a sharp geometric trailing edge have been previously been shown to have greater time-averaged drag but lower lift and pitching moment than airfoils with a blunt trailing edge $[15,18]$. This work expands on this finding to demonstrate that the unsteady airloads are generally greater for airfoils with a sharp trailing edge and, perhaps more importantly from a vibrations standpoint, become periodic at much lower angles of attack.

\section{Acknowledgements}

This work was supported by the U.S. Army/Navy/NASA Vertical Lift Research Center of Excellence with Mahendra Bhagwat serving as Program Manager and Technical Agent, grant number W911W6-11-2-0012. The authors would like to recognize Ignacio Andreu, Luke Smith, and Dr. Bharath Govindarajan for their contributions to this work. The authors would also like to thank Dr. Joesph Milluzo, Dr. David Miklosovic, and the wind tunnel technicians at the United States Naval Academy. 


\section{References}

[1] J. F. Manwell, J. G. McGowan, A. L. Rogers, Wind Energy Explained, John Wiley \& Sons, Ltd., 2002.

[2] A. Pellegrino, C. Meskell, Vortex shedding from a wind turbine blade section at high angles of attack, Journal of Wind Energy and Industrial Aerodynamics 121 (2013) 131-137.

[3] J. G. Leishman, Principles of Helicopter Aerodynamics, 2nd Edition, Cambridge Univ. Press, 2006.

[4] A. Bagai, Aerodynamic design of the $x 2$ technology demonstrator ${ }^{\mathrm{TM}}$ main rotor blade, in: $64^{\text {th }}$ Annual Forum of the AHS, 2008.

[5] M. Potsdam, A. Datta, B. Jayaraman, Computational investigation and fundamental understanding of a slowed uh-60a rotor at high advance ratios, in: $68^{\text {th }}$ Annual Forum of the AHS, 2012.

[6] A. Datta, H. Yeo, T. R. Norman, Experimental investigation and fundamental understanding of a full-scale slowed rotor at high advance ratios, Journal of the American Helicopter Society 58 (2).

[7] B. Berry, I. Chopra, Wind tunnel testing of an instrumented rotor at high advance ratio, in: $56^{\text {th }}$ AIAA/ASCE/AHS/ASC Structures, Structural Dynamics, and Materials Conference, no. AIAA 20150950, Kissimmee, FL, 2015.

[8] G. Bowen-Davies, I. Chopra, Aeromechanics of a slowed rotor, in: $56^{\text {th }}$ AIAA/ASCE/AHS/ASC Structures, Structural Dynamics, and Materials Conference, no. AIAA 2015-0951, Kissimmee, FL, 2015.

[9] A. H. Lind, L. R. Smith, J. I. Milluzzo, A. R. Jones, Reynolds number effects on sharp and blunt trailing-edge airfoils in reverse flow, Journal of Aircraft (submitted).

[10] R. F. Anderson, The aerodynamic characterisitcs of six commonly used airfoils over a large range of positive and negative angles of attack, NACA TN 397 (1931).

[11] A. Pope, The forces and pressures over an naca 0015 airfoil through 180 degrees angle of attack, Georgia Institute of Technology.

[12] C. C. Critzos, H. H. Heyson, R. W. Boswinkle, Jr., Aerodynamic characteristics of naca 0012 airfoil section at angles of attack from $0^{\circ}$ to $180^{\circ}$, NACA TN 3361 (1955).

[13] R. E. Sheldahl, P. C. Klimas, Aerodynamic characteristics of seven symmetrical airfoil sections through 180-degree angle of attack for use in aerodynamic analysis of vertical axis wind turbines, Tech. rep., Sandia National Laboratories (March 1981).

[14] W. Timmer, Aerodynamic characterisitics of wind turbine blade airfoils at high angles-of-attack, in: European Academy of Wind Energy TORQUE 2010, Crete, Greece, 2010.

[15] A. H. Lind, J. N. Lefebvre, A. R. Jones, Time-averaged aerodynamics of sharp and blunt trailing edge static airfoils in reverse flow, AIAA Journal 52 (12) (2014) 2751-2764. 
[16] J. M. Rainbird, J. Peiro, J. M. R. Graham, Blockage-tolerant wind tunnel measurements for a naca 0012 at high angles of attack, Journal of Wind Engineering and Industrial Aerodynamics 145 (2015) $209-218$.

[17] S.-C. Yen, Aerodynamic performance and shedding characteristics on a swept-back wing, Journal of Maring Science and Technology 19 (2) (2011) 162-167.

[18] A. H. Lind, A. R. Jones, Vortex shedding from airfoils in reverse flow, AIAA Journal 53 (9) (2015) $2621-2633$.

[19] R. E. Akoury, M. Braza, Y. Hoarau, J. Vos, G. Harran, A. Sevrain, IUTAM Symposium on Unsteady Separated Flows and Their Control, Vol. 14, Springer Netherlands, 2009, Ch. Unsteady Flow Around a NACA0021 Airfoil Beyond Stall at 60 deg Angle of Attack, pp. 405-415.

[20] M. J. Smith, N. D. Liggett, B. C. G. Koukol, Aerodynamics of airfoils at high and reverse angles of attack, Journal of Aircraft 48 (6) (2011) 2012-2023.

[21] F. H. Norton, D. L. Bacon, Pressure distribution over thick aerofoils-model tests, NACA TR 150 (1922).

[22] A. Naumann, Pressure distribution on wings in reverse flow, NACA TM 1011 (1942).

[23] D. G. Mabey, Some aspects of aircraft dynamic loads due to flow separation, Progress in Aerospace Sciences 26 (1989) 115-151.

[24] C. H. K. Williamson, R. Govardhan, Vortex-induced vibrations, Annual Review of Fluid Mechanics 36 (2004) 413-455.

[25] C. F. Coe, J. A. Mellenthin, Buffeting forces on two-dimensional airfoils as affected by thickness and thickness distribution, NACA RM A53K24 (1954).

[26] K. Mulleners, A. L. Pape, B. Heine, M. Raffel, The dynamics of static stall, in: $16^{\text {th }}$ International Symposium of Laser Techniques to Fluid Mechanics, Lisbon, Portugal, 2012.

[27] A. Fage, F. C. Johansen, On the flow of air behind an inclined flat plate of infinite span, Proceedings of the Royal Society of London 116 (773) (1927) 170-197.

[28] G. B. McCullough, D. E. Gault, Examples of three representative types of airfoil-section stall at low speed, NACA TN 2502 (1951)

[29] J. M. Chen, Y.-C. Fang, Strouhal numbers of inclined flat plates, Journal of Wind Engineering and Industrial Aerodynamics 61 (1996) 99-112.

[30] J. R. Calvert, Experiments on the low-speed flow past cones, Journal of Fluid Mechanics 27 (1967) 273-289. 
[31] J. E. L. Simmons, Similarities between two-dimensional and axisymmetric vortex wake, Aeronautical Quarterly 26 (1977) 15-20.

[32] I. V. Kolin, V. G. Markov, T. I. Trifonova, Shukhovtsov, Hysteresis in the static aerodynamic characterisitcs of a curved-profile wing, Technical Physics 49 (2) (2004) 124-127.

[33] H. Hu, Z. Yang, H. Igarashi, Aerodynamic hysteresis of a low-reynolds-number airfoil, Journal of Aircraft 44 (2007) 2083-2086. 